\title{
Human remains identification using micro-CT, spectroscopic and A.I. methods in forensic experimental reconstruction of dental patterns after concentrated acid significant impact
}

\author{
Andrej Thurzo 1,7,8*, Viera Jančovičová ${ }^{2}$, Miroslav Hain ${ }^{3}$, Milan Thurzo ${ }^{4}$, Bohuslav Novák ${ }^{1}$, Helena Kosnáčová ${ }^{8,9}$, \\ Viera Lehotská ${ }^{10}$, Norbert Moravanský ${ }^{5,7}$ and Ivan Varga ${ }^{6}$
}

1 Department of Stomatology and Maxillofacial Surgery, Faculty of Medicine, Comenius University in Bratislava, 81250 Bratislava, Slovakia; bohuslav.novak@fmed.uniba.sk

2 Department of Graphic Arts Technology and Applied Photochemistry, Institute of Natural and Synthetic Polymers, Faculty of Chemical and Food Technology, Slovak University of Technology in Bratislava, Radlinského 9, 81237 Bratislava, Slovakia; viera.jancovicova@stuba.sk

3 Institute of Measurement Science, Slovak Academy of Sciences, Dúbravská cesta 9, 84104 Bratislava, Slovakia; umerhain@savba.sk

4 Department of Anthropology, Faculty of Natural Sciences, Comenius University in Bratislava; Slovakia, milanthurzo@gmail.com

5 Institute of Forensic Medicine, Faculty of Medicine Comenius University in Bratislava, Sasinkova 4, 81108 Bratislava, Slovakia; norbert.moravansky@forensic.help

6 Institute of Histology and Embryology, Faculty of Medicine, Comenius University in Bratislava, 81372 Bratislava; Slovakia; ivan.varga@fmed.uniba.sk

7 forensic.sk Institute of Forensic Medical Expertise, expert institute, Boženy Němcovej 8, 81104 Bratislava, Slovakia; info@forensic.sk

8 Department of Simulation and Virtual Medical Education, Faculty of Medicine, Comenius University in Bratislava, Sasinkova 4, 81272 Slovakia; Andrej@Thurzo.sk

9 Department of Genetics, Cancer Research Institute, Biomedical Research Center, Slovak Academy of Sciences, Bratislava, Dubravska Cesta 9, 84505 Slovakia; helena.svobodova@fmed.uniba.sk

10 2nd Department of Radiology, Faculty of Medicine, Comenius University in Bratislava, Heydukova 10, 81250 Bratislava, Slovakia; viera.lehotska@ousa.sk

* Correspondence: Andrej.Thurzo@fmed.uniba.sk; Tel.: (+421 903110 107)

Simple Summary: Modern technologies now offer new approaches in forensic human skeletal identification affecting fields of forensic anthropology, forensic odontology and forensic radiology. One of the most resilient remnants preserved in human remains, are teeth. These usually contain interventions made by a dentist. These interventions, typically dental fillings, represent so called "dental patterns" which can be reconstructed even from a nearly destroyed human remains. These reconstructions can be matched to previous dental records and used for identification. Dental patterns have superior distinctive characteristics. They can survive even morphological destruction of original human tissues including bones and teeth - for example action of concentrated acid. With digitalization, micro- and also cone beam computed tomography, machine learning and spectroscopic methods, the reconstruction of dental patterns can today provide more than only digital two-dimensional photo-matching. This article introduces a new methodological perspective with the utility of above-mentioned technologies as well as the context of unpublished morphological changes of human bones, teeth and dental fillings accompanying the action of $75 \%$ sulfuric acid. The article introduces the current state of the art on the roles of forensic anthropology and forensic dentistry when applied to the identification from dental patterns.

\section{Abstract:}

(1) Human teeth are the most resilient tissues in the body. However, exposure to concentrated acids might lead to their obliteration, thus making human identification difficult. Teeth often contain dental restorations from materials that are even more resilient to acid impact. This paper introduces novel method of 3D reconstruction of dental patterns as a crucial step for digital identification with dental records.; (2) With combination of modern methods of Micro-Computed Tomography, Cone 
Beam Computed Tomography, Attenuated Total Reflection in conjunction with Fourier-Transform Infrared Spectroscopy and Artificial Intelligence Convolutional Neural Network algorithms, the paper presents the way of 3D dental pattern reconstruction and human remains identification. Research studies morphology of teeth, bone and dental materials (Amalgam, Composite, Glass-ionomer cement) under different periods of exposure to 75\% sulfuric acid; (3) Results reveal significant volume loss in bone, enamel, dentine and as well glass-ionomer cement. Results also reveal significant resistance of composite and amalgam dental materials to sulfuric acid impact, thus serving as strong parts in the dental pattern mosaic. Paper also introduces probably first successful artificial intelligence application in automated forensic CBCT segmentation.; (4) Interdisciplinary cooperation utilizing mentioned technologies can solve problem of human remains identification with 3D reconstruction of dental patterns and their 2D projections over existing ante-mortem records.

Keywords: Dental Pattern; Forensic Anthropology; Forensic Odontology; Forensic Dentistry; Forensic Radiology; Forensic Chemistry; Forensic science; Machine Learning; Identification; Dental materials; Acid solution; Dissolution; Sulfuric acid; Dentition; Teeth; Acid degradation;

\section{Introduction}

\section{Human identification from obliterated remains - the problem}

Teeth, as the most resilient structures of the body, resist destruction better than bones. However, they are not as resilient as some dental materials. These can resist not only mechanical forces, but also the heat of fire, decay, or other means of destruction including acids. Acid destruction prevails over other ways of destroying parts of the body in order to prevent identification. Acids can dissolve DNA, metals, the whole body and liquefy even bones and teeth. Sulfuric, hydrochloric, nitric and their combinations are among the most frequently used acids for this purpose in forensic history. Only a very few materials of a specific composition, most often plastics-composites, can usually withstand this total destruction. 3D scanning and 3D reconstructions help to identify these microscopic elements. These might be root or composite fillings, plastic nails or other composite materials.

Among the first cases of "Acid Bath Murderers" is the British assassin John George Haigh, who in the 1940s dissolved at least six of his victims, in barrels filled with sulfuric acid. He said the victims had completely dissolved in about two days. He also said he had to leave the room because the fumes were unbearable. Special plastic containers are used to store these aggressive substances, and a conventional bathtub, as is often visualized in films, is not a reliable container as it is itself subject to corrosion.

During the police arrest, John George Haigh said, "Mrs. Durand-Deacon no longer exists, I destroyed her with acid. You can't prove murder without a body." He was arrested after the last of the murders. The Sussex workshop he rented did not have a floor drain. He therefore disposed the remains by pouring the container onto a pile of rubble at the back of the garden. Examination of the area by pathologist revealed $12.7 \mathrm{~kg}$ of human, part of the human foot, gallstones and plastic part of the denture, which was later identified during the trial as Ms. Durand-Deacon. He later confessed to another eight murders, in which he destroyed the body in a sulfuric acid bath. Haigh, whose motive was money, was finally hanged for his crimes in 1949.

Reconstruction and identification of human remains after an extensive destruction, for example in acids, might be extremely difficult even with utilization of the most advanced technologies. Current technologies have introduced new methodological perspectives in forensic human skeletal identification. Solution to a problem of reconstruction and identification of such altered human remains might be supported in multidisciplinary combination of state-of-the-art digital methodologies connecting the fields of forensic anthropology; forensic dentistry; forensic radiology; forensic chemistry and possibly with implementation of artificial intelligence. 
The solution to a problem of otherwise unidentifiable human remains lies in threedimensional (3D) digital reconstruction of most resilient parts of human remains - teeth. With their very specific alterations supported with medical records they provide a possibility of reconstruction of - so called dental patterns. Dental patterns show substantial variability not only between age groups within the same year of birth, but also between different years for the same age group. Anthropological and dental elements, supported by medical records, offer the crucial distinctiveness and power of discrimination necessary for forensic human identification [1-3].

Forensic dentistry and forensic anthropology require interdisciplinary knowledge, since information obtained from the remains can significantly contribute to identify an individual or provide information needed in a legal process. Furthermore, the data obtained from dental patterns can considerably narrow the search range of an individual and play a key role in the victim identification process following mass disasters or a criminal disposal of a body [2].

Forensic dentistry is a specialist branch of forensic sciences It is primarily concerned with the use of teeth and surrounding oral structures for identification in a legal context. Various forensic odontology techniques help in the identification of the human remains in incidents such as terrorists' attacks, airplane, train and road accidents, fires, mass murders, and natural disasters such as tsunamis, earth quakes and floods, and many others. Most frequently they face the problem of a single victim comparison between post-mortem findings and ante-mortem dental records. Forensic dentistry (odontology) brings knowledge of orofacial structures, their variation, and the implications of dental treatment to the identification process. Forensic dentistry has well-established roots dating back more than 2,000 years. Originally, it was not a scientific discipline requiring advanced training. There have been many technological advances in the science of dentistry since the $4^{\text {th }}$ century. Modern imaging techniques and methods involving dental diagnostics and care have advanced the profession into the modern era. This article communicates an interdisciplinary combination of digital photography, cone-beam computed tomography (CBCT) [4], micro computed tomography (micro-CT) [5,6], advanced Artificial Intelligence (AI) algorithms like 3D Convolutional Neural Network (CNN) algorithms [7-9] and Attenuated Total Reflection (ATR) in conjunction with Fourier-Transform Infrared Spectroscopy (FTIR)[10,11].

These advances offer the forensic expert a useful armamentarium permitting even archived cases to be revisited. The disciplines within the forensic dentistry and anthropology have expanded beyond dental identifications to include recognition and reporting of child and elder abuse, age assessment, and bitemark analysis and many more [1,2,12-14].

Identification of an unidentified body is rarely a straightforward path. The investigation and identification are frequently established on a multidisciplinary cooperation and integrated, multiagency coordinated approach. Forensic Dentistry has been, for a long time, a reliable discipline in the process of human identification. The combination of all available dental characteristics, leads to a unique dental pattern, that offer an individualizing and differentiating potential with regard to the personal identification process. For example, dental fractures or abrasions can be produced during life or post-mortem. These can be differentiated. Ante-mortem abrasions may be indicative of bad habits (bruxism) or abrasion with ceramic dental restoration (crown or bridge) on antagonist teeth[15].

Sometime the reconstruction of these patterns is difficult and modern digital technologies need to be implemented. Digital 3D reconstruction supports the effective match to the pre-mortem dental records. In this logic, human identification through comparative dental evidence does not inevitably have to meet a minimum number of resemblances (i.e., concordant points or matching evidence), but rather the significant number or quality of points of concordance between ante-mortem and post-mortem records (with no unexplainable differences). Further, the process of human skeletal identification must provide conclusions about the degree of certainty or probability of identification. New artificial intelligence algorithms can enhance methods of estimations already practiced in human anthropology like estimation of age or diet [16-18]. At this current point, new forensic 
identification methodologies, which are based on the quantification of evidentiary value for forensic radiologic comparisons of dental patterns, are excellent. In cases of missing dental therapeutical alterations on the preserved teeth and bones, further studies and documentation of population frequencies for skeletal and dental traits/characteristics are desired. Additional research in this area will contribute significantly to Forensic Dentistry, Forensic Anthropology and Forensic Radiology by facilitating the use of anatomical diversity for identification and can be based in a very near future on advanced artificial intelligence algorithms $[1,2,7,9,19,20]$.

\section{Tissue destruction in acids}

Teeth are the most durable structures of a body resisting various means of destruction even more than skeletal tissue. Above-described concept of dental patterns takes advantage of the fact, that therapeutical alterations of teeth and surrounding tissues (tooth fillings, crowns, bridges, implants etc.) can be even more resilient. Commercially available acids can be used to obliterate the human remains in effort to make its identification impossible. Criminals may be use Sulfuric acid, $\mathrm{HNO}_{3}$, or $\mathrm{HCl}$ to hinder the person identification; still, the individuals can be identified using resilient dental patterns. These frequently persist even after exposure to the concentrated acids [21,22].

Teeth can serve as a reliable tool in identification even after exposure to concentrated sulfuric acid $\left(\mathrm{H}_{2} \mathrm{SO}_{4}\right)$. In absence of matchable dental records, they frequently serve for age estimation or sex determination of the victim [23].

In a "mafia" - type crime cases, forensic experts frequently face situations where criminals were trying to destroy a cadaver by immersing it in acids. In these situations, dental patterns are represented as residues possible for identification. This paper approximates processes morphologically and chemically happening to bone and dental structures when exposed to $75 \%$ sulfuric acid. Experience of other scientists shows that teeth are completely dissolved after 14 hours of immersion in $37 \%$ solution of hydrochloric acid, while at 90 hours in $96 \%$ sulfuric acid. Even after 90 hours in $96 \%$ sulfuric acid, the destruction of the samples was still incomplete. In nitric acid the teeth undergo a complete dissolution in 12 hours, and in 17 hours in aqua regia (chloroazotic acid-hydrochloric/nitric acid 1:3). Despite exposure to acids the Mazza et al. 2005 were able to recognize the characteristic morphological features of dental tissues and structures up until the advanced stages of degradation [24].

Dental materials frequently resist decomposition and high temperatures and are among the last ones to disintegrate after death. The principles of the dental identification lie in the fact that no two oral cavities are alike and the teeth and their therapeutical modifications (dental patterns) are unique to an individual. The dental evidence of the deceased recovered from the scene of crime/occurrence is compared with the ante-mortem records for identification. Resistance of teeth to acid exposure is remarkable among all body tissues. Dental features such as tooth morphology, variations in shape, size, number of cusps, restorations, pathologies, missing teeth, wear patterns, malocclusions like crowding of the teeth, colour and position of the tooth, rotations and other peculiar dental anomalies give every individual a unique identity - dental pattern [25]. 


\section{The technology of cone-beam computed tomography}

In this paper, it is described a combination of three technologies with additional presentation of artificial intelligence algorithm for automated cone-beam computed tomography (CBCT) segmentation and digital photo matching. This novel approach for reconstruction and matching of dental patterns depends on CBCT, micro-CT and spectroscopic methods. These can be widened for spectroscopic and micro-CT evaluation of other dental materials in the future.

When the problem is described as morphological destruction of tissues and experts face necessity to match it to the dental records a CBCT is the most typical available computed tomography available. It is used primarily in maxillofacial applications. CBCT may also be very useful in some forensic contexts, offering numerous advantages for post-mortem forensic imaging including a good resolution for skeletal forensic radiology, relatively low cost and simplicity. CBCT may be a beneficial and accessible alternative in many cases [26].

Identification of unknown skeletal remains using dental comparison is well established in the forensic setting. Most frequently a two-dimensional (2D) intraoral photo or 2D x-ray image from dental records are used for matching. In different levels of morphological destruction of skeletal human remains due to various reasons including acids, a reconstruction of dental patterns is a turntable approach.

The identification technique can be a time and resource consuming if many individuals need to be identified in a short time. Using a CBCT unit, protocols have been developed to reproducibly and reliably reformat $\mathrm{CBCT}$ volumes. The reformatted images can be compared directly with conventional digital images from the same anatomic area. Images derived from $\mathrm{CBCT}$ volumes are similar enough to conventional dental radiographs to allow 2D dental forensic comparison and identification. CBCT offers a superior option over multi-slice CT scanners for this purpose [27].

As the 2D reconstruction was appropriate for matching two 2D radiographic images, it was facing a struggle to find the appropriate projection. As the dental records are typically in 2D format, it is sufficient; albeit it is not necessary to give up of the advantage of $3 \mathrm{D}$ reconstructed matching to the $2 \mathrm{D}$ image. The reconstruction of skeletal remains using conventional $\mathrm{CBCT}$ provide accurate three-dimensional reconstructions, which demonstrate its reliable use as a forensic tool [28].

Post-mortem dental examinations play an important role in the individual identification. In forensic dentistry, in the first step of individual identification is based on a traditional visual comparison of the ante-mortem dental record and dental radiographs with those obtained by postmortem examinations. Digitization in forensic odontology started more than 30 years ago. It enabled a new era of digital processing, nowadays leading to smart AI algorithms performing trillions of matching sequences per second, albeit yet to be established as an accurate and reliable methodologies by scientific research. Forensic methods with digital technology are nowadays more accepted than in the past, when were considered as expensive, requiring special equipment and the cost of other components. Further research is currently being conducted and progress is made every day in the field of forensic odontology along with the progression of general digital technology [29].

In regards to the current dental records, these are changing too. Together with progress in digital diagnostics, we witness more frequent availability of 3D digital records (CBCT from dental diagnostics in orthodontics or CT and nuclear magnetic resonance images from other medical examinations). Forensic odontology has long been an essential discipline in the post-mortem identification of human remains and it adapts too. Much of forensic dental identification is based upon the comparison of one set of records with another. Aside from the documented dental or other medical records, dental casts and digital intraoral scans and photographs, radiographs are one of the main sources of evidence. These are being compared with contemporaneous images taken of the subject. In the process of reconstruction of dental patterns, these examinations are performed on the remains [30]. 
CBCT was found to be more accurate compared to orthopantomography (OPG) in dental anatomy evaluation. Small field of view of CBCT centered on the mandibular angle of a young patient is an accurate and safe method for dental age estimation around the age of 18 [31].

Forensic radiology exists in a specific field of medical imaging technologies that are utilized to assist the forensic experts. Cone Beam Computed Tomography (CBCT) is frequent in forensic odontology for image analysis examinations. Through CBCT radiology, evolutionary forensic odontology has been developed extensively in many applications. The frequent role of forensic witnesses ' in analysis of bite marks, investigation of trauma cases, and determination of age, sex and race are often multidisciplinary approach implementing skillsets of forensic anthropologist, radiologists and dentists. The advantages of digital CBCT radiographs help significantly in identification checks in forensic, especially in skeletal and odontology cases [4].

\section{The technology of micro computed tomography}

The micro computed tomography (micro-CT) within forensic practice remains an emerging technology, principally due to its present limited availability to forensic practitioners. This paper puts it in the context, within the presented complex analysis, in effort to reconstruct dental patterns; facing the problem of morphological destruction of tissues. Its value is the significantly higher level of detail when compared to CBCT. Micro-CT provides information about characteristic details accompanying the process of destruction as well as the potential details of dental procedures performed. For example; about technique of the dentist in cavity preparation or other micro details not identifiable on CBCT.

The practical role of micro-CT in forensic expertise is to bring more details in comparison to other x-ray examination of the remains. Its implementation in the 3D dental pattern reconstruction is to enhance forensic investigation [5].

In the application of scientific human skeletal variation in medico-legal matters, the virtual anthropology is the modern method implemented to examine skeletal remains. Digital imaging methods are used in many areas of forensic dentistry. We shall understand, that these dental records were created on a living tissue and thus with limited radiation dose and limited accuracy. On the contrary the post-mortem records have no relevant limitations of this kind and can provide unprecedented 3D information in significantly higher detail. Among all digital imaging modalities in dentistry, digital periapical radiography is the most frequently used; albeit with limited area covered and limited information value. However, new contemporary imaging techniques like micro-computed tomography (Micro-CT) can be used in post-mortem analysis of the remains. The accuracy and reliability of enamel and dentin thickness measurement with Micro-CT was researched. Comparison was made between periapical radiographs and Micro-CT methods and there were differences evaluated between measurements by periapical radiography and Micro-CT methods. Micro-CT showed the highest accuracy. Dentin and enamel measurements by Micro-CT was highly accurate and reliable as in the conventional method. Micro-CT evaluations are recommended for implementation in the future anthropological studies [32].

Age estimation is a notable aspect of forensic science. Accurate age estimation methods are required for an increasing number of situations such as unidentified body remains, refugees and asylum seekers without proof of identification or other people with question of a threshold age that has arisen for legal reasons. It is known that pulp chamber volume of the maxillary second molars has the largest correlation coefficient with age. Also, Micro-CT can be implemented for this task to provide accurate information about the volume proportion of the pulp chamber [33].

Micro-CT can be forensically utilized for analysis of detailed marks like saw marks created on human bone. Micro-CT as a powerful and reliable imaging method for the measurement of saw mark properties [34]. 
The advanced Artificial Intelligence (AI) algorithms proved as useful tools in processing digital data and soon will subdue the area of automatized assessment of age, sex, ethnicity, microfractures, or dental pattern identification and comparison. This is inevitable and will provide forensic expertise with unprecedented efficient analytic tools [7].

AI can be understood as a branch of computer science that describes the research and development of simulated human intelligent behaviour in machines. This involves researching methods that enable a computer to develop behaviour similar to human thinking and work independently on problems. The introduction of cone beam computed tomography (CBCT) to dental-maxillofacial radiology in the late 1990s has transformed the way the profession analyzes the oral and maxillofacial complex. Dental providers, who were previously limited to 2D images of truly 3D anatomical structures, suddenly had the ability to visualize the hard tissues of the head and neck in all the desired planes as well as in 3D reconstructions. CBCT scans may reveal hidden pathology and incidental findings of clinical or forensic significance located in structures outside a dentist's conventional area of focus. The frequency of incidental findings identified by CBCT has been reported to be as high as 3.2 findings per scan. The most frequent incidental findings identified by CBCT were malignancy, maxillary sinus pathology and ameloblastoma. [9].

This paper introduces first practical forensic implementation of AI algorithm used for auto-segmentation of the mandible, mandibular teeth and their sockets as well as the canal of mandibular nerve.

The AI algorithms will bring unprecedented analytical tools; however, they need to be trained first. For this a vast amount of data is necessary. In field of forensic radiology, a specific algorithm can be created which further helps to identify distinct microscopic characteristics forming an array of specific features in the 3D space that would represent a new form of AI - identified dental patterns hardly comprehensible with a human brain. AI is advancing in the field of dental radiology and will bring a paradigm shift within the next decade [7,9,35-46].

Dental records play an important role as well for AI in modern forensic identification. Post-mortem dental patterns reconstructed possibly with AI algorithms will be compared to considerable amounts of digital records of variable quality, covered anatomical region, time of origin or type of diagnostic technology. Technologies used today providing data that can contribute to final completion of mosaic are: 3D intraoral optical scan, 3D CBCT scan of the skull, 3D nuclear magnetic resonance scan of the head, 3D lidar face scan, 3D ultrasonographic scan of submandibular region, 2D dental local x-ray scans or orthopantomography scan and many more. Most of them are dismissed today as they are too difficult to implement and research has not proved them to be accurate for the final matching, yet. This wide variety of possibly hundreds medical and dental records are as well randomly distributed in time from childhood until the final days. Ability of AI to simulate aging, based on big data trained networks, this can help adapt these records in time and incorporate them valuably into the final mosaic of the reconstructed dental pattern[7].

With narrowed focus, currently, dental findings and teeth conditions are recorded in a dental chart and compared with those of ante-mortem records. However, this is a physically and mentally laborious task, especially in large scale disasters. AI can automate this process. Unlike the conventional methods, AI methods are advantageous in obtaining high classification accuracy without the need for precise tooth segmentation. The AI tooth automated classification method can be useful in automatic filing of dental charts for forensic identification [47].

Currently the number of studies reporting the use of AI in the forensic aspects is relatively low. Nevertheless, AI developments in prosthodontics are demonstrating its application for automated diagnostics, as a predictive measure, and as a classification or identification tool. In the future, AI technologies will likely be used for collecting, processing, and organizing patient-related datasets to provide patient-centered, individualized dental treatment [48].

A wide range of enablers for implementing AI in the dental diagnostics are known (e.g., the chance for significantly higher diagnostic accuracy, a reduced workload, even 
more comprehensive reporting and better patient-provider communication). Opinion leaders in the field will consider these aspects to foster implementation of AI in dentistry [49].

A novel AI system based on deep learning methods, which was used for the mandible auto-segmentation presented in this paper, was scientifically evaluated in the past (Ezhov et al. 2021). This research determined its real-time performance of CBCT imaging diagnosis of anatomical landmarks, pathologies and clinical effectiveness when used by dentists in a clinical setting. The system consists of five modules: ROI-localization-module (segmentation of teeth and jaws), tooth-localization and numeration-module, periodontitis-module, caries-localization-module, and periapical-lesion-localization-module. These modules use convolutional neural network based on state-of-the-art architectures. These are considered with highest potential for CBCT scan processing [7]. In total, 1,346 CBCT scans were used to train the modules. After annotation and model development, the AI system was tested for diagnostic capabilities of the Diagnocat AI system. 24 dentists participated in the clinical evaluation of the system. Thirty CBCT scans were examined by two groups of dentists, where one group was aided by Diagnocat and the other was unaided. The results for the overall sensitivity and specificity for aided and unaided groups were calculated as an aggregate of all conditions. There was a statistically significant difference between the groups $(\mathrm{p}=0.032)$. This study showed that the proposed AI system significantly improved the diagnostic capabilities of participating dentists [8].

The success of the artificial intelligence (AI) system in dental implant planning using three-dimensional cone-beam computed tomography (CBCT) images has been published recently. Further development of AI systems and their utilization in future implant planning will both facilitate the work of dentists and will be a support mechanism in implantology practice to physicians [50].

AI has proven efficient as well in detecting impacted third molars on cone-beam computed tomography scans. Diagnostic performance of AI application evaluating the impacted third molar teeth in CBCT images was clinically evaluated. The AI application showed high accuracy values in the detection of impacted third molar teeth and their relationship to anatomical structures [51].

The role of $\mathrm{AI}$ in the identification of periapical pathosis on CBCT scans is impressive as well. Diagnostic performance of an AI system based on the deep convolutional neural network method to detect periapical pathosis on CBCT images has been evaluated in the past. Research of Orhan et al. 2020 of the deep convolutional neural network system showed the system was successful in detecting teeth and numbering specific teeth. Only one tooth was incorrectly identified. The AI system was able to detect 142 of total 153 periapical lesions. The reliability of correctly detecting a periapical lesion was $92.8 \%$. The deep convolutional neural network volumetric measurements of the lesions were similar to those with manual segmentation. There was no significant difference between the two measurement methods ( $p>0.05$ ). Volume measurements performed by humans and by AI systems were comparable to each other. AI systems based on deep learning methods can be useful for detecting periapical pathosis on CBCT images for clinical application [52].

AI is currently underestimated in the implementations in forensic anthropology, dentistry, radiology and chemistry. It will undoubtedly shift the paradigm in this area $[7,35,38,45,53-59]$.

\section{The technology of Infrared spectroscopy for spectroscopic forensic evaluation}

Forensic chemistry is the application of chemistry and its subfield, forensic toxicology, in a legal setting. A forensic chemist can assist in the identification of unknown materials found at a crime scene. In the focus of this article the bone and tooth tissues including dental fillings undergo particular chemical changes when exposed to $75 \%$ sulfuric acid. Specialists in this field use a wide array of methods and instruments to help identify chemical composition of unknown substances. One these technologies implemented in this article them is Attenuated Total Reflection (ATR) in conjunction with Fourier- 
Transform Infrared Spectroscopy (FTIR). This is the final pillar of a novel hybrid and complex forensic approach for reconstruction of dental patterns, introduced in this paper.

FTIR has been used extensively for chemical characterization of mineralized tissues in the past few decades. FTIR is an ideal technique to analyze chemical structural properties of materials, since the frequencies of several vibrational modes of organic and inorganic molecules are active in the infrared. FTIR methodology, in conjunction with the attenuated total reflection (ATR) sampling mode, is suitable particularly for characterization of enamel, dentin and bone tissues. These are composed of a mineral and an organic phase. The mineral phase is characterized essentially as nonstoichiometric substituted apatite, being the carbonate and phosphate spectral peaks the main representative of these phase. Organic matrix of the post-eruptive enamel is very small ( $\sim 1 \%$ weight). The dentin and bone organic phases are mainly composed of type I collagen that appears as spectral bands of amide I, amide II, amide III bands. Moreover, synthetic apatite materials are being designed for total or partial replacement, restoration or augmentation of these biological tissues with FTIR assistance [60].

Due to a deficit of reliable and accurate methods, determination of the post-mortem interval of human skeletal remains is one of the most important and challenging tasks in forensic medicine. Various studies of the changes to bone chemistry were using FTIR spectroscopy in conjunction with other spectroscopic methods. For example, research published with Wang et al. 2017 evaluated paired bone samples collected from 56 human corpses that were buried (in soil) and unburied (exposed to the air) for intervals between 76 and 552 days. This study illustrates the feasibility of utilizing FTIR spectroscopy and chemometrics as an attractive alternative for estimating post-mortem interval of human remains and the great potential of these techniques in real forensic cases with natural conditions [61].

Another remarkable utilization of ATR-FTIR was published with Chophi et al. in 2020. Human lips are often covered with lipsticks layer. Due to its prevalence, it can be frequently encountered at the crime scene as evidence. ATR-FTIR analyses of such exhibits can provide evidence to link the suspect with the victim or with the crime scene or support the victim identification. In this study, 38 different red shade lipsticks of 20 different manufacturers were analyzed using ATR-FT-IR (attenuated total reflectance-Fourier transform-infrared) spectroscopy. Chemometric methods: principal component analysis and linear discriminant analysis, applied to provide objective interpretation of results resulted in $100 \%$ discriminating power and $81.48 \%$ correct classification respectively. The effects of different substrates on lipstick analyses, lipstick wear time for one day, and exposure to different environmental conditions were also studied. More research is required for linking lipstick smudges on different substrates with their source of origin using chemometric methods [62]. However, this application indicates the path of enhancing the dental pattern reconstruction protocol with dental material chemical changes as a vital part.

Many dental materials might contain gelatin which is a protein derived either from porcine, bovine or other animal sources. ATR-FTIR can be utilized as a reliable tool to detect gelatin in dental materials and other pharmaceuticals [63]. ATR-FTIR can be utilized to as a simple, sensitive and non-destructive technique for characterizing dental enamel erosion. Even though various techniques are available to assess enamel erosion in vitro, a simple, non-destructive method with sufficient sensitivity for quantifying dental erosion is required in forensic aspects. ATR-FTIR spectroscopy is potentially advantageous over the microhardness test as a simple, non-destructive, sensitive technique for the quantification of enamel erosion [64].

Dental materials exposed to chemically of otherwise destructive force undergo dynamic process that might stop on a particular level. Even aging of the dental material might render chemical changes. For example, in methacrylate peak determination, ATRFTIR can be utilized to investigate polymerization of dental methacrylate mixtures. Investigation of polymerization kinetics using ATR-FTIR systems is common in many dental studies. However, peak selection methods to calculate monomer-polymer conversion can 
vary, consequently affecting final results. ATR-FTIR can reliably assess polymerization of methacrylate and is therefore suitable for forensic analysis as well [65].

ATR-FTIR mapping can be applied to screening in situ bone and teeth preservation in several modern and archaeological samples. It allows multi-scale and multi-technique approaches with the potential to identify the better-preserved areas in fossils. Bones and teeth are highly hierarchically structured and heterogeneous materials, and post mortem processes can reinforce this heterogeneity. It is therefore important to consider this heterogeneity to better understand diagenetic processes. In research of Lebon et al. 2014, ATRFTIR mapping method was applied to several archaeological samples. ATR-FTIR mapping provided spatially resolved information on alteration state of mineral and organic matter. This technique allowed to describe the spatial distribution of organic and mineral matter preservation in unheated Paleolithic bones (Bize-Tournal, France) characterized by a better preservation in the center of the cortical bone. Spatial variations in the chemical composition of an archaeological heated bone (Abri Pataud, France) compared to a modern reference suggested taphonomical uptake of carbonate in the most external part. FTIRATR is a powerful tool that allows identification and characterization of local heterogeneities in bone preservation. This technique opens new prospects to reconstruct the taphonomical history of ancient samples [10].

ATR-FTIR is ideal for evaluation of surface alteration of human tooth enamel subjected to acidic and neutral 30\% hydrogen peroxide in terms of chemical structure, mechanical property, surface morphology and tooth color. The study of Sun et al. 2011, suggested that neutral $30 \%$ hydrogen peroxide had the same efficiency in tooth bleaching and it caused less deleterious effects on enamel than acidic 30\% hydrogen peroxide [66].

ATR-FTIR analysis was also used in estimation of possible differences in the chemical composition and relaxation of orthodontic aligner materials [67].

A forensic investigation of single human hair fibers using FTIR-ATR spectroscopy and chemometric evaluation was performed and published by Barton in 2011. Human hair fibers are ubiquitous in nature and are found frequently at crime scenes often as a result of exchange between the perpetrator, victim and/or the surroundings. Therefore, hair fiber evidence can provide important information for crime investigation. For human hair evidence, the current forensic methods of analysis rely on comparisons of either hair morphology by microscopic examination or nuclear and mitochondrial DNA analyses. Unfortunately, in some instances the utilization of microscopy and DNA analyses are often not feasible. Barton has presented a protocol as a systematic method to rapidly investigate unknown or questioned single human hair using FTIR-ATR spectra from different genders and racial origin, including fibers of different cosmetic treatments. Unknown or questioned spectra were first separated on the basis of chemical treatment i.e., untreated, mildly treated or chemically treated, genders, and racial origin i.e., Asian, Caucasian and African-type. The methodology has complemented the current forensic analysis methods of fiber evidence (i.e., Microscopy and DNA), providing information on the morphological, genetic and structural levels [68].

Human and non-human identification of unknown skeletal remains is of great importance in forensic and anthropologic contexts. However, the traditional morphological methods for bone species identification are subjective or time-consuming. With utilization of FTIR spectroscopy and spectroscopic methods forensic experts are able to determinate the spectral variances between human and non-human (i.e., pig, goat, and cow) bones. FTIR spectroscopy is a reliable tool to assist in bone species determination and also has great potential in real forensic cases [69].

Utilization of ATR-FTIR spectroscopy in separating forensic, WWII, and archaeological human skeletal remains is also frequent. As ATR-FTIR spectroscopy is proven as a fast and accessible, minimally or non-destructive technique which provides information on physiochemical characteristics of analyzed materials. In forensic and archaeological sciences, it is commonly used for answering numerous questions, including the archaeological or forensic context of the human skeletal remains. Results of research published by Leskovar et al. 2019, showed that employing whole spectral domains works best for the 
separation of archaeological, WWII, and forensic samples, even with samples of highly variable origin [70].

Aim, highlights and principal conclusion

Aim of this article is to introduce a novel multidisciplinary approach for forensic human identification from acid-obliterated remains based on dental pattern reconstruction.

Highlights of the article are:

- Human mandible with post-mortem treated teeth was degraded in $75 \%$ sulfuric acid and accompanying morphological and chemical changes were documented.

- $\quad$ CBCT and Micro-CT technologies were used for 3D reconstruction of dental patterns

- $\quad$ ATR-FTIR spectroscopy was utilized to investigate the of human skeletal remains.

- Advanced AI-CNN algorithm was utilized for automated mandible segmentation.

- This research provides unprecedented 3D morphological set of four stages of degradation of human mandibular bone and teeth presented on five different regions/samples.

The principal conclusion of the article is that combined multidisciplinary effort of experts from forensic dentistry, forensic anthropology, forensic radiology and forensic chemistry can reconstruct dental pattern from even acid-obliterated human remains and use it for successful identification.

\section{Materials and Methods}

The primary working hypothesis for this research was that dental restorations can resist the degradation effect of $75 \%$ sulfuric acid more that dental tissues.

The secondary hypothesis was premising that $3 \mathrm{D}$ reconstructed dental patterns can be utilized for matching with $2 \mathrm{D}$ dental records represented with intraoral digital photo and OPG scan.

The bone of human mandible with teeth (Figure $\mathbf{1} \mathbf{a}, \mathbf{b}$ ), described in this article, was donated from Institute of Forensic Medical Expertise - Forensic.sk. It originated as a random anonymous finding during excavation of a canceled cemetery and was intended for disposal. It was found without a remaining skeleton and collected by forensic experts for scientific research more than 20 years ago. 


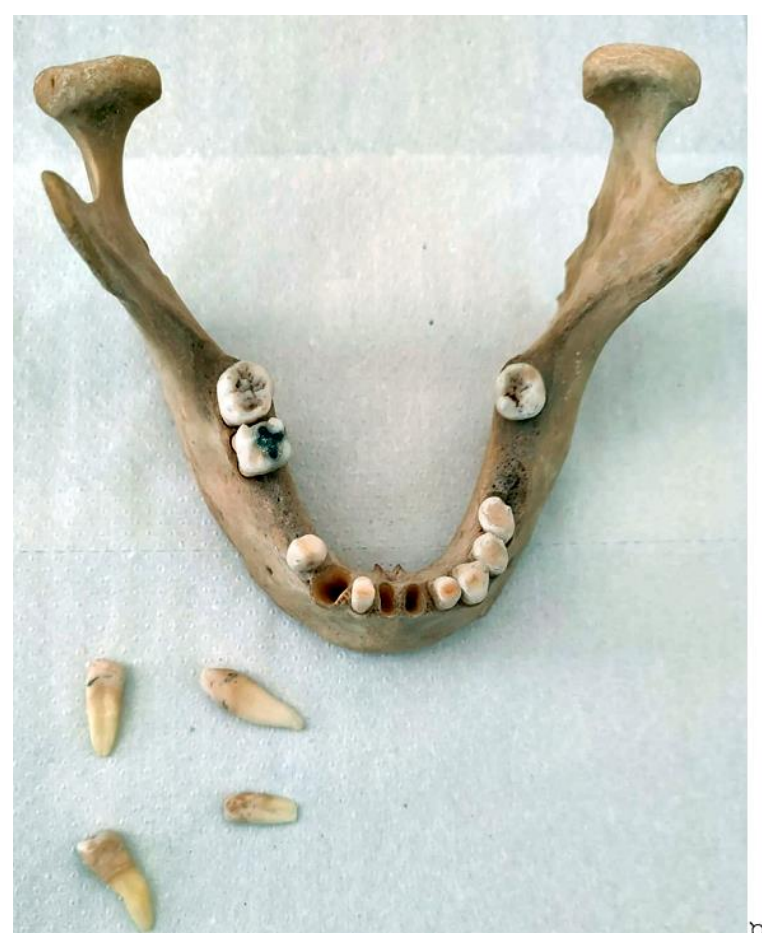

(a)

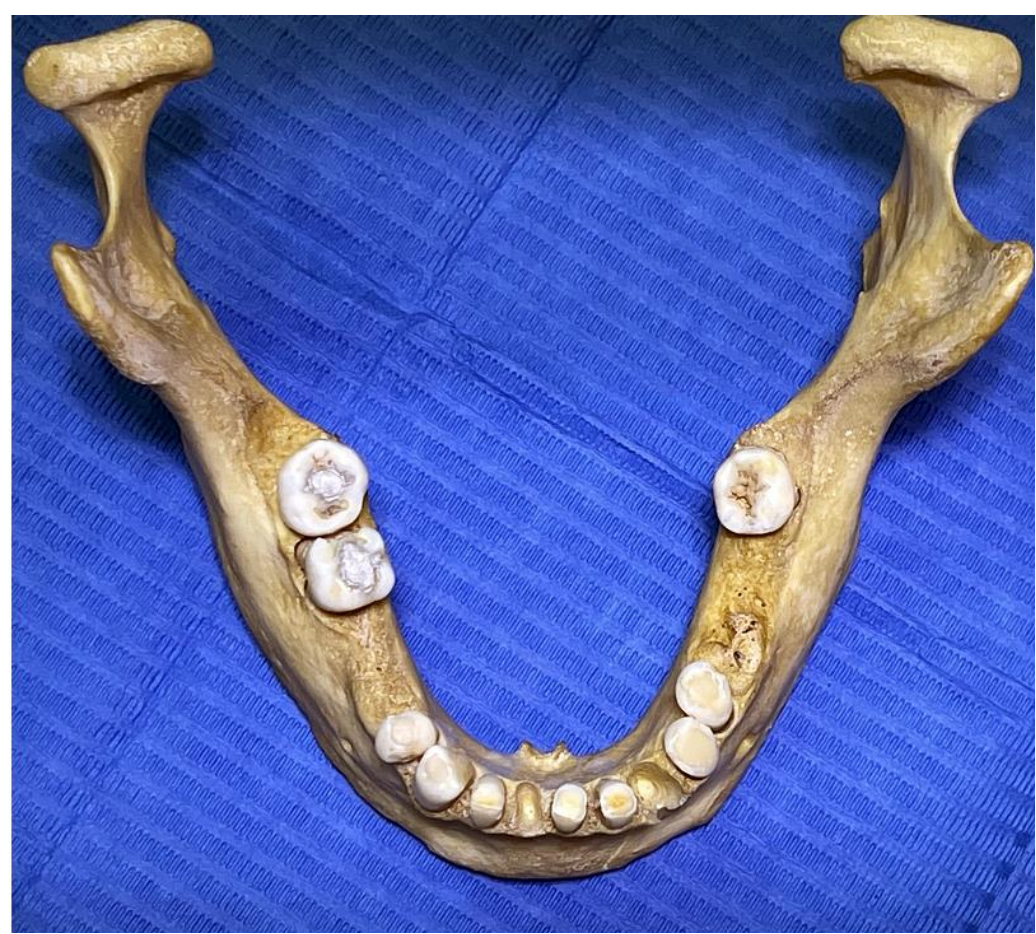

(b)

Figure 1. The human mandible with teeth: (a) Before creating dental preparations; (b) After preparations and CBCT scan.

Digital optical scanning of the original mandible was performed with iTero Element intraoral scanner and exported to STL (Figure $2 \mathbf{a}, \mathbf{b}$ ). Models from this output were compared to segmented models from CBCT to prove this optional step as a valid procedure. Only one initial scan was created and was sufficiently accurate in comparison to segmented CBCT mandibular surface (Figure $\mathbf{2} \mathbf{b}$ ).

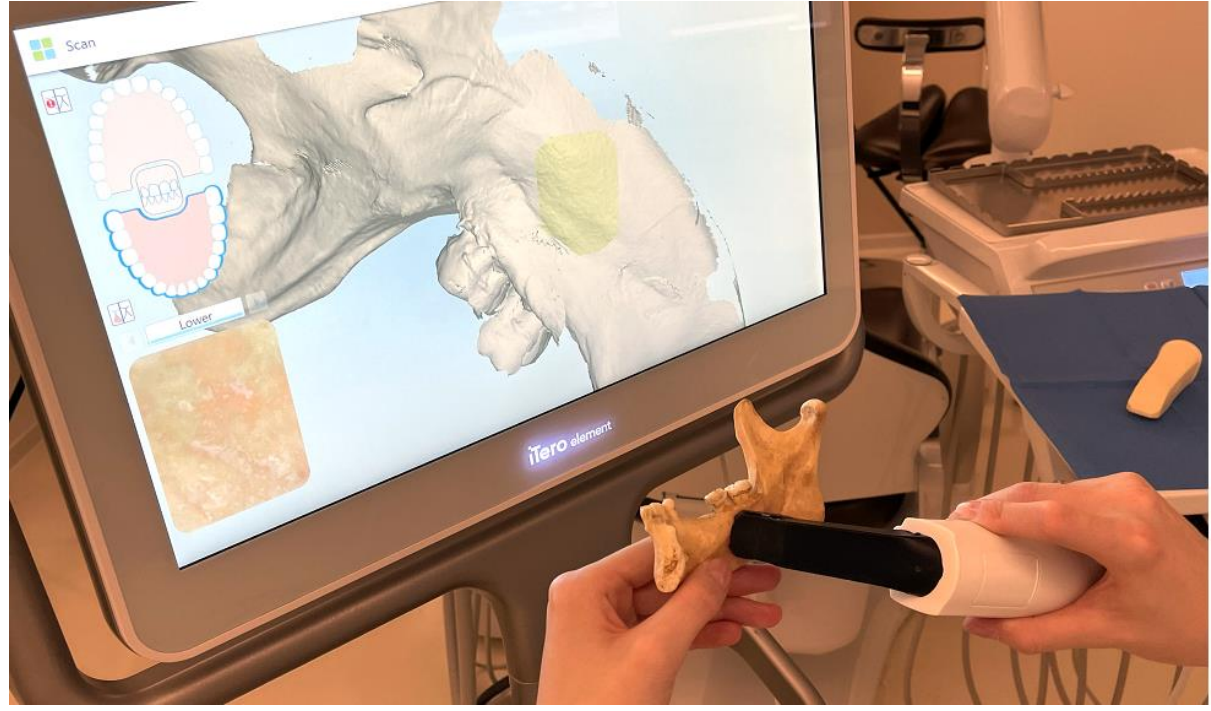

(a)

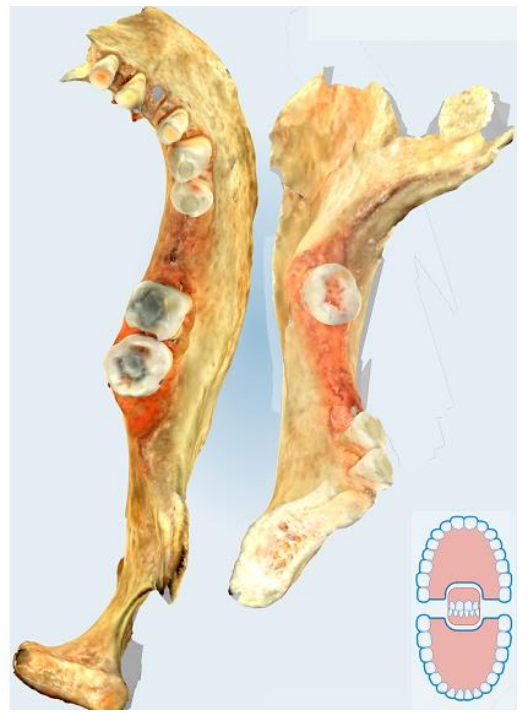

(b)

Figure 2. Optical scanning of the mandible: (a) iTero Element intraoral scanner and other scanners are commonly available; (b) PLY/STL export for comparison to the CBCT segmented models. This approach confirmed sufficient accuracy of the surface scan, albeit remains optional, as the surface scan has no morphological information about the inner structure.

In three pairs of teeth, dental fillings of a special shape were created with three different dental materials (Figure 3 a). Older dental filling was removed. The objective was 
to create a plane surface that would be extending the surrounding enamel surface to allow contact with the FTIR sensor (Figure $3 \mathbf{b}$ ). Later the objective of FTIR examination of dental fillings has been abandoned due to possible breakage of the whole tooth in later stages of the degradation process. Three pairs of tooth fillings were created from the following dental materials:

1. Dental amalgam - Ana 2000 Capsules Non-Gamma Two, Extra High Copper (Contains: 43\% Silver, 26.1\% Copper, 30.8\% Tin)

2. Glass ionomer - GC FUJI IX GP wear-resistant multipurpose (Contains: Powder: 95\% Fluro alumino silicate glass, 5\% Polyacrylic acid powder; Liquid: 40\% Polyacrylic acid and tartaric acid, 50\% Distilled water, 10\% Polybasic carboxylic acid)

3. Dental composite - Neo Spectra ST (Contains: Methacrylate modified polysiloxane, Dimethacrylate resins, fluorescent pigment, UV stabilizer, Camphorquinone, Ethyl-4 (dimethylamino)benzoate, Bis (4-methyl-phenyl) iodonium hexafluorophosphate, Barium-aluminum-borosilicate glass, Ytterbium fluoride, Iron oxide pigments and titanium oxide pigments according to shade). Prime \& Bond Universal were used as adhesive system in preparation with composite.

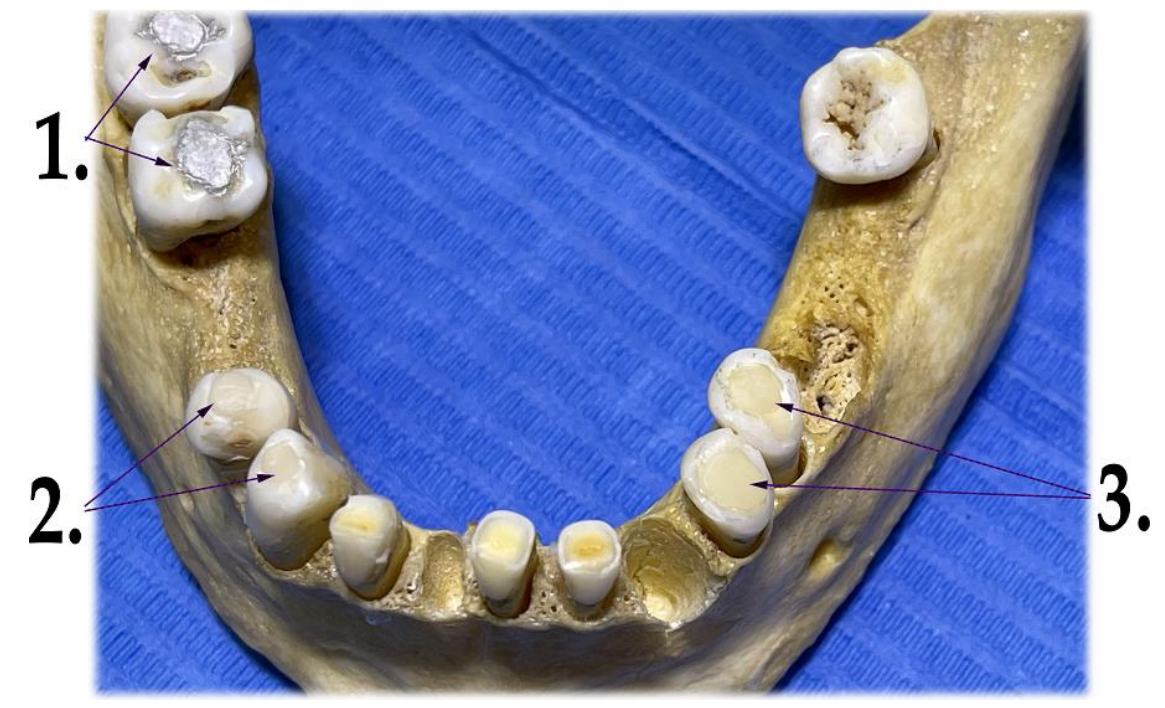

(a)

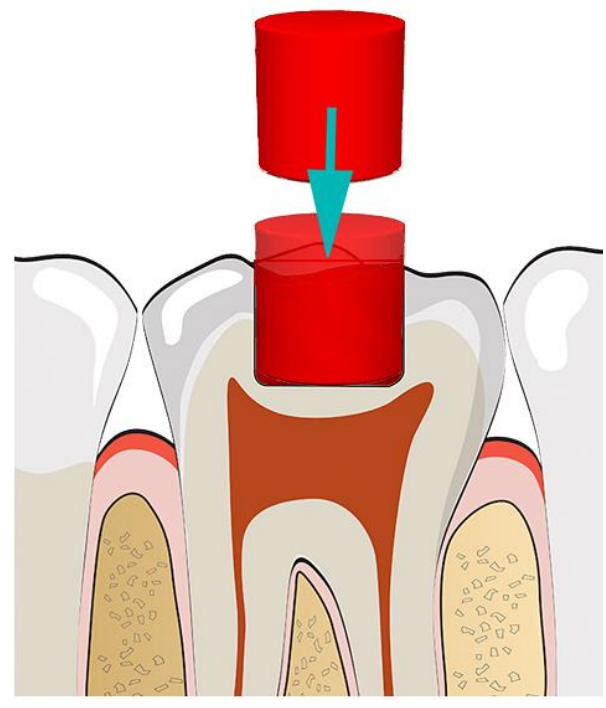

(b)

Figure 3. Dental fillings made on the teeth post-mortem: (a) Three dental materials were used in dental restorations: (1) Amalgam Extra High Copper Non-Gamma Two - Ana 2000 Capsules, (2) Glass ionomer - GC FUJI IX GP wear-resistant, (3) Photo-composite methacrylate resin - Neo Spectra ST (Methacrylate modified polysiloxane); (b) The filling was created with occlusal part above the enamel surface with horizonal plane with intention to support the contact with the FTIR sensor. This objective has been abandoned due to high probability of tooth destruction in later stages of the aciddegradation process.

CBCT used for scanning (Figure $4 \mathbf{a}, \mathbf{b}$ ) was performed with Planmeca ProMax 3D Mid CBCT with the following parameters:

- Panoramic exposure with following settings and values: 2D Panoramic, Standard, Patient size $\mathrm{M}=$ Medium-sized adult, $67 \mathrm{kV}, 11 \mathrm{~mA}, 15 \mathrm{~s}$

- 3D exposure was performed with following settings: CBCT Volume Ø100x100 in High Definition (HD) mode, voxel size $150 \mu \mathrm{m}$

- Two pairs of OPG and CBCT were created. First scan before teeth preparative treatment and final scan after preparation. 


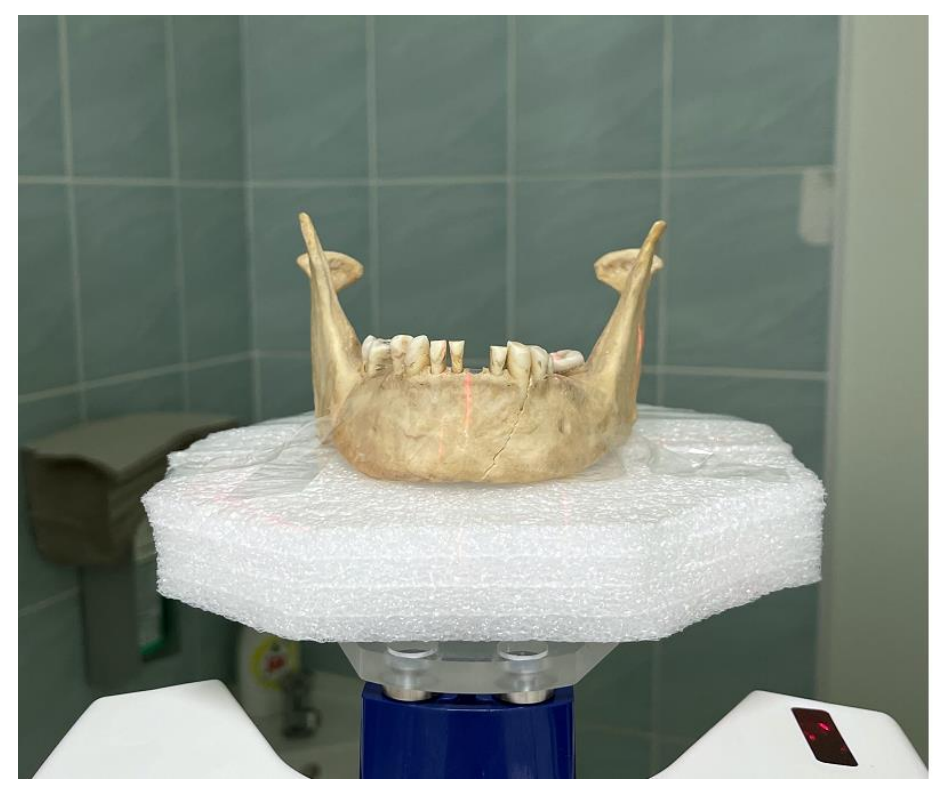

(a)

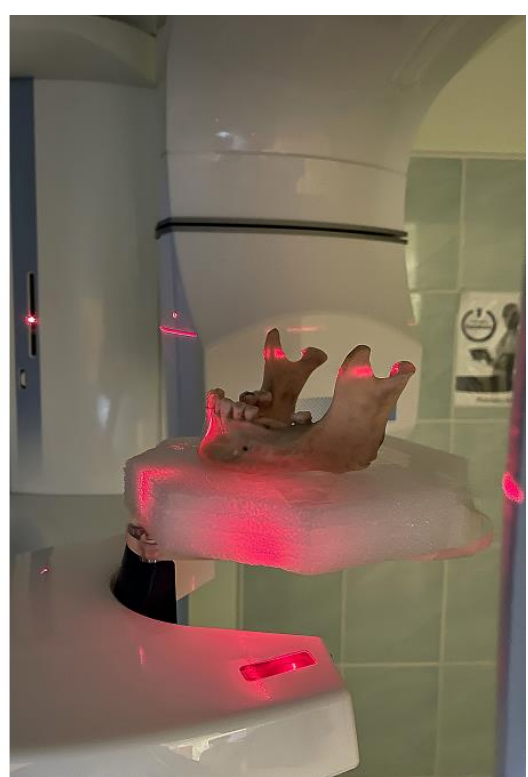

(b)

Figure 4. CBCT scanning with Planmeca ProMax 3D Mid CBCT: (a) Positioning of the mandible consisting from two fragments and stabilization of teeth in the sockets; (b) OPG and CBCT scans were created before and after dental restorations were made.

The final CBCT scan with custom post-mortem dental preparation was subjected to manual and as well a novel advanced AI CNN algorithm for segmentation of teeth and jaw.

For manual segmentation was used full version of software Invivo dental 6.5 (Anatomage, Inc., Santa Clara, CA, USA). For AI automated segmentation the mandibular segment, teeth, dental sockets and canal for mandibular nerve were segmented with Diagnocat on-line program (LLC "DIAGNOCAT" Moscow, Russia) (Figure 5). This solution processed the CBCT scan of the mandible within five minutes and created STL segments of described anatomical structures with the use of convolutional neural network [8,5052]. The STL segments were downloaded from this program and stored on computer.

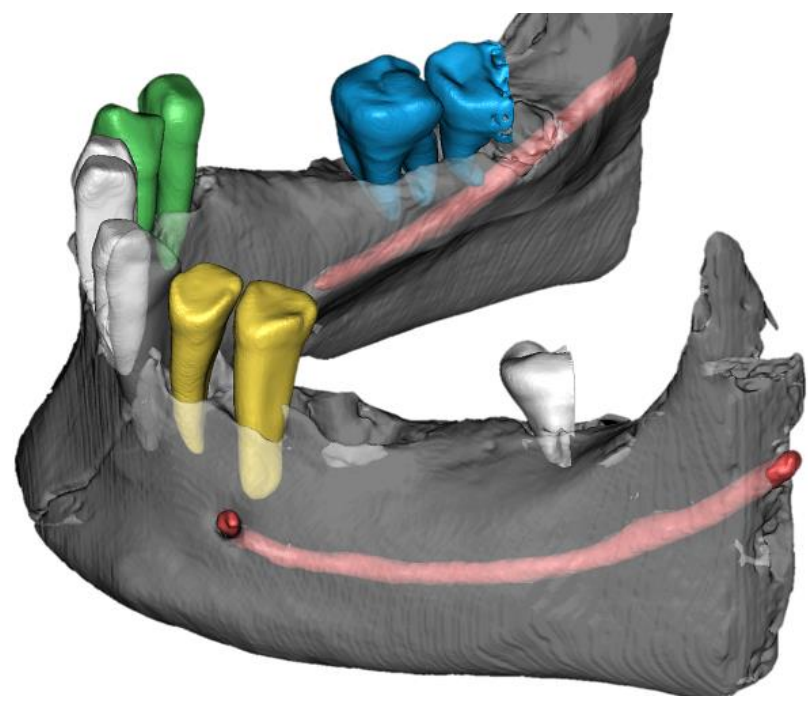

(a)

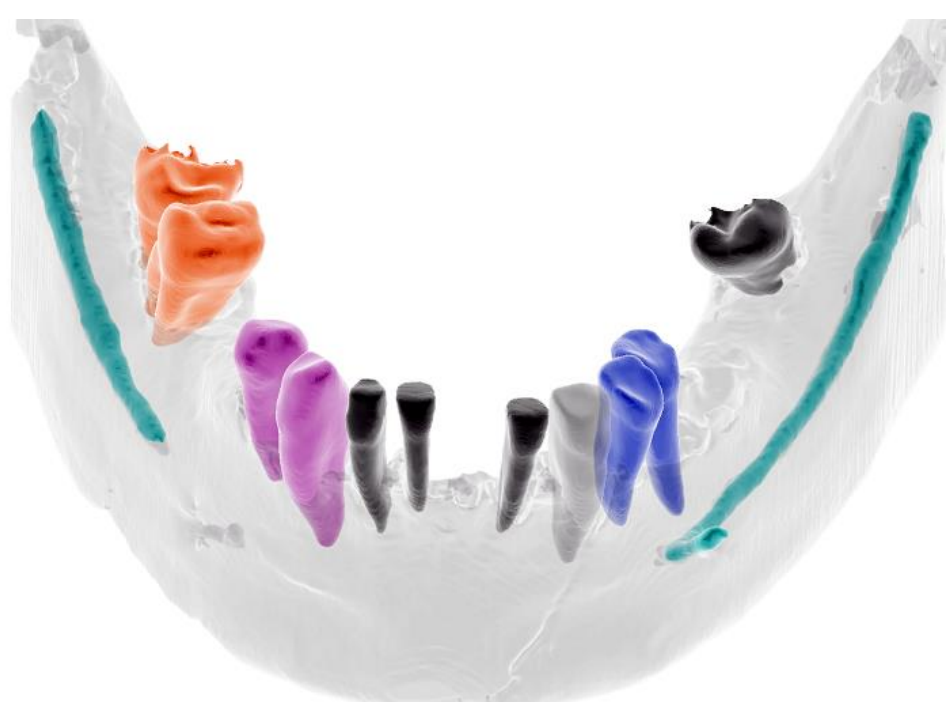

(b)

Figure 5. AI automated segmentation of the mandible with on-line tool Diagnocat: (a) Separate segments of teeth, bone, and nerve canal cam be visualized with different colors, opacity and exported as separate STLs; (b) Scan of teeth without preparations shows unfinished recognition of the right mandibular canal and cut of distal parts of wisdom teeth. 
After CBCT scanning the mandible has been cut into five segments (Figure 6) and scanned initially with X-ray micro-CT.

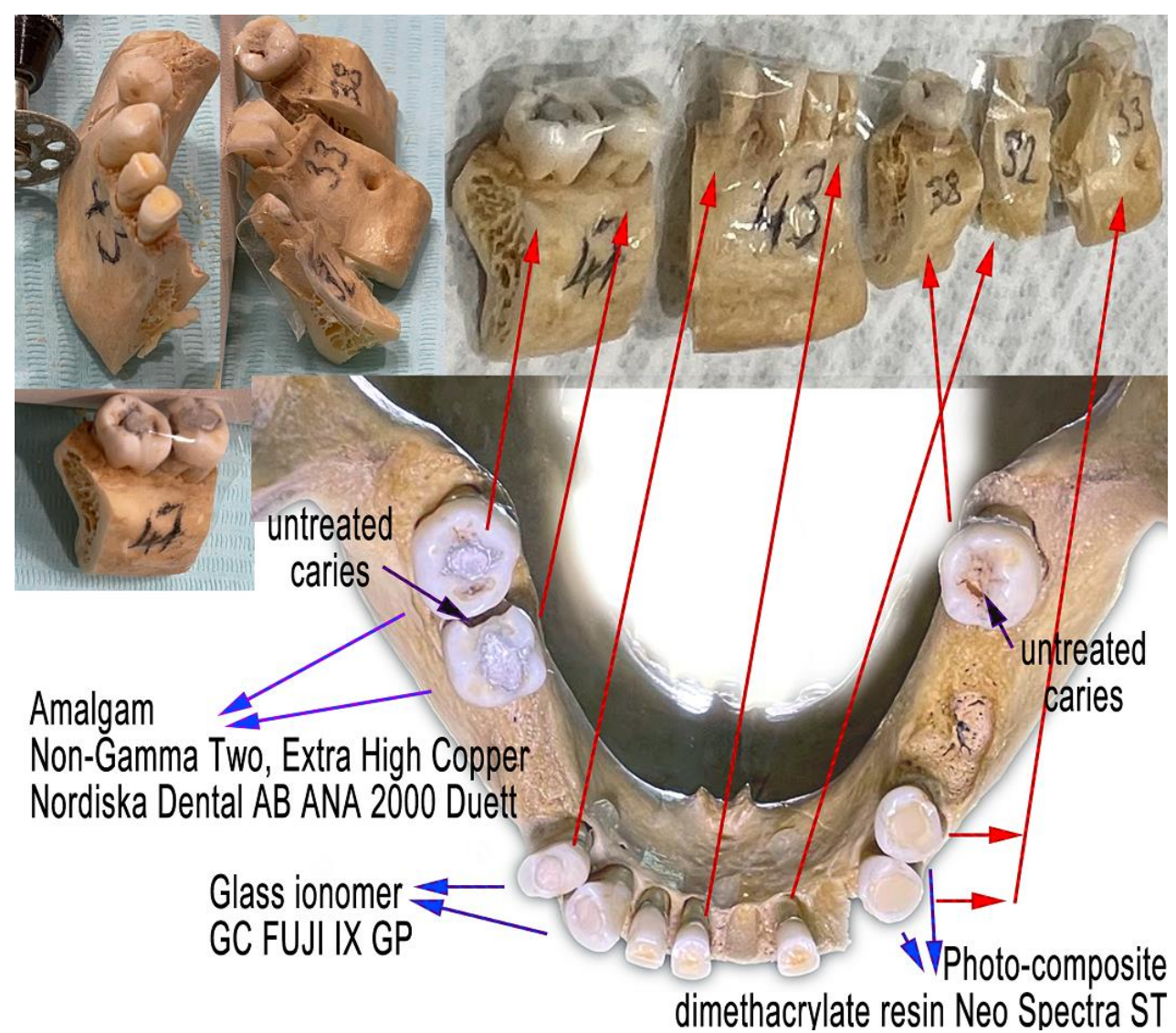

Figure 6. The mandible was cut into five segments labeled $(47,43,38,32$ and 33) to allow repeated sequences of sulfuric acid bath, triple washing, drying, photographing and Micro-CT scanning.

Micro-CT scans were created using microtomography system Nanotom180 (GE Phoenix). The X-ray tube was fitted with a tungsten target and micro-focusing mode M0 was used. For standard micro-CT scan of a tooth the following settings were used: accelerating voltage $\mathrm{U}=150 \mathrm{kV}$, beam current $\mathrm{I}=80 \mu \mathrm{A}$, detector sampling time $\mathrm{t}=750 \mathrm{~ms}$. Voxel size was $20 \mu \mathrm{m}$ and totally $1,800 \mathrm{X}$-ray projections were recorded during one $\mathrm{CT}$ scan. For filtering of outgoing X-ray beam a $0.2 \mathrm{~mm} \mathrm{Cu}$ plate was used.

For micro-CT scans of a tooth with amalgam filling different settings were used: accelerating voltage $\mathrm{U}=170 \mathrm{kV}$, beam current $\mathrm{I}=90 \mu \mathrm{A}$, detector sampling time $\mathrm{t}=500 \mathrm{~ms}$ and pixel binning $2 \times 2$ was applied. For filtering of outgoing X-ray beam a $0.5 \mathrm{~mm}$ Sn plate was used. The 3D volume reconstruction was done using Phoenix datos $\mid x$ CT software using Feldkamp filtered back-projection algorithm. The 3D data sets were rendered and segmented using VGStudio MAX 2.1 software. For the segmentation of volume data, the region growing method was mostly used along with the opening/closing and erosion/dilation image processing techniques (Figure $\mathbf{7} \mathbf{a}, \mathbf{b}, \mathbf{c}$ ). The segmented 3D model of the preserved dental filling is a crucial part of dental pattern and will be used in matching to different 2D projections from pre-mortem dental records (Figure $7 \mathrm{c}$ ). 


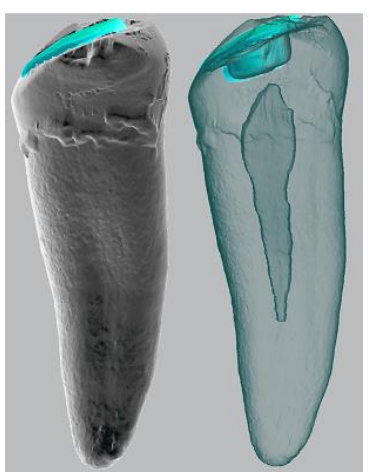

(a)

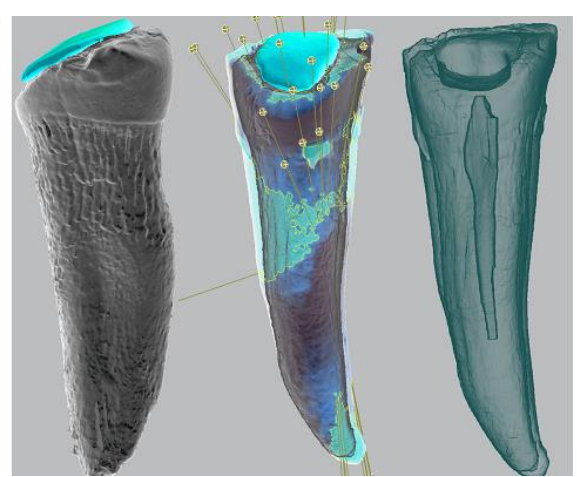

(b)

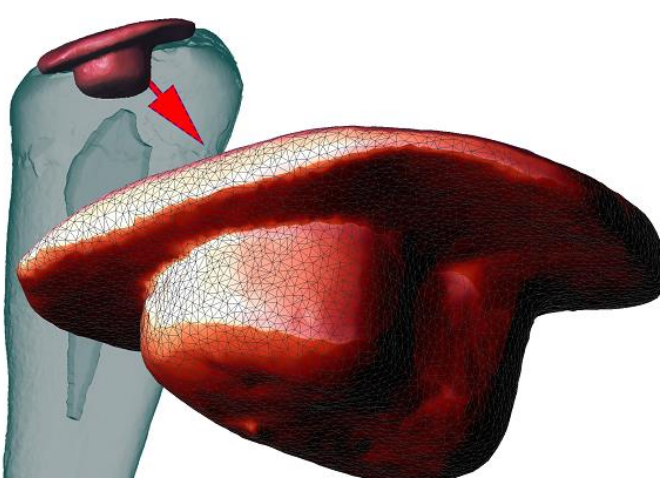

(c)

Figure 7. Outputs from micro-CT segmentation: (a) Original segment of tooth 34 before acid bath; (b) segments of tooth and dental filling after 96 hours in acid bath; (c) 3D reconstructed dental filling for future projections over various 2D (typically) diagnostic pre-mortem dental record (x-rays, digital intraoral photography).

The five segments of the mandibular bone with treated and untreated teeth were exposed to $75 \%$ sulfuric acid in sequential exposition after 2, 6 and 24 hours. After each exposition the specimens were washed and dried and a Micro-CT scan was performed on every sample. There were 4 Micro-CT scans for each sample and each scan was segmented into bone part and tooth part as a separate STL (Figure 8). Set of output STLs were made publicly available on-line. Visualizations of micro-CT segmentation outputs of all 5 samples are summarized in detail in Appendix section of this article as well as digital photo documentation.

Samples of bones and teeth were submerged in the $75 \%$ sulfuric acid. This concentration was chosen after consideration together with expert chemists in the scientific team and also upon the field experience of forensic experts in the team in regards to their recommendations and other aspects that shall not be publicly disclosed. There is known a substantial difference of $\mathrm{H}_{2} \mathrm{SO}_{4}$ dynamics in the process of bone degradation dependent on its concentration. More aggressive degradation take place with less concentrated $\mathrm{H}_{2} \mathrm{SO}_{4}$.

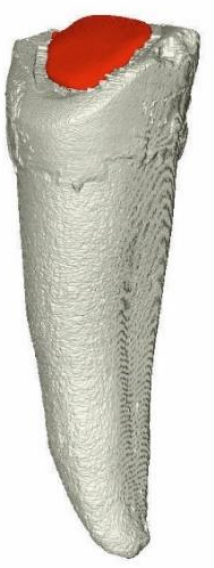

$\mathrm{Oh}$

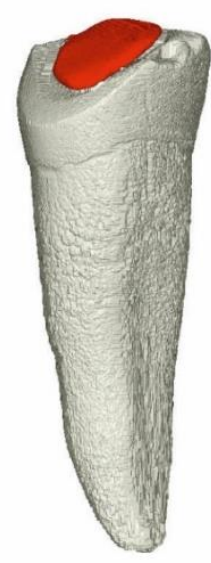

$6 \mathrm{~h}$

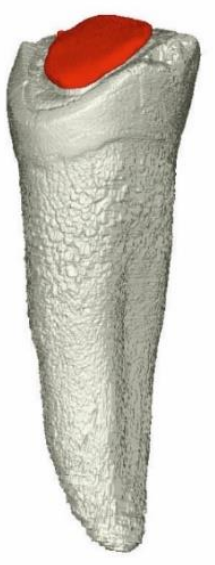

$24 \mathrm{~h}$

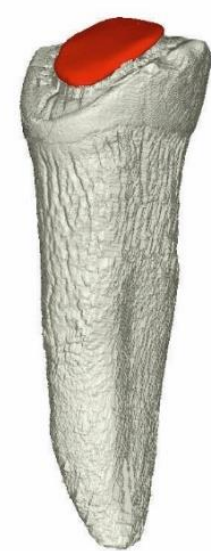

$96 \mathrm{~h}$

Figure 8. There were 4 micro-CT scans made for each sample and each scan was segmented into three parts: bone, tooth, and in case of treated tooth, also with dental material part. On this picture is preparation with photo-composite resin on tooth 34 from the segment labeled " 33 " after exposure to $75 \%$ sdulfuric acid after different times of exposure. It is obvious the composite filing is not losing the volume in the acid bath in contrast to remaining tooth structures. 
After timed exposure, all five samples were taken out from the acid bath and were washed three times in deionized water and then dried in a multifunctional oven APT Line Series FED with the regulation R 3.1 by Fisher Scientific at the temperature of $40{ }^{\circ} \mathrm{C}$ for 60 minutes. At this time of intermission, micro-CT scan was performed after 2, 6, 24 and 96 hours. As well the ATR-FTIR spectra were measured, however, only after 2, 6 and 24 hours. The ATR-FTIR was not measurable after 96 hours of acid exposure as the samples were too fragile and ATR-FTIR measurement would represent a significant risk of the sample mechanical destruction.

Subsequently, after the measurements the samples were re-immersed in fresh / new sulfuric acid $\left(75 \% \mathrm{H}_{2} \mathrm{SO}_{4}\right)$ and the whole procedure was repeated.

Method of ATR FTIR spectroscopy was performed with the following setting. Attenuated Total Reflectance (ATR) mode FTIR spectra of bone and teeth samples were acquired using FTIR spectrophotometer Varian Excalibur Digilab FTS 3000MX, (Palo Alto, CA, USA), equipped with the ATR adapter with diamond crystal. The measurement range was from 4000 to $600 \mathrm{~cm}^{-1}$; resolution $4 \mathrm{~cm}^{-1}$, sensitivity 8; 30 scans. The background was air. Three different spectra in different places were measured, averaged and evaluated using Origin (version 8.5, MicroCal) software.

\section{Results}

\subsection{Descriptive morphological evaluation based on micro-CT analysis}

Significant volume loss was observed on segments of bone, teeth and glass-ionomer cement. Insignificant volume loss was detected on amalgam $(<0.1 \%)$. No volume loss was detected on photo-composite dental material. Volumetric changes after 6, 24 and 96 hours are visualized in the (Figure 9) and detailed in Table 1.

Tooth, bone, photocomposite, glass ionomer cement, and amalgam volume loss

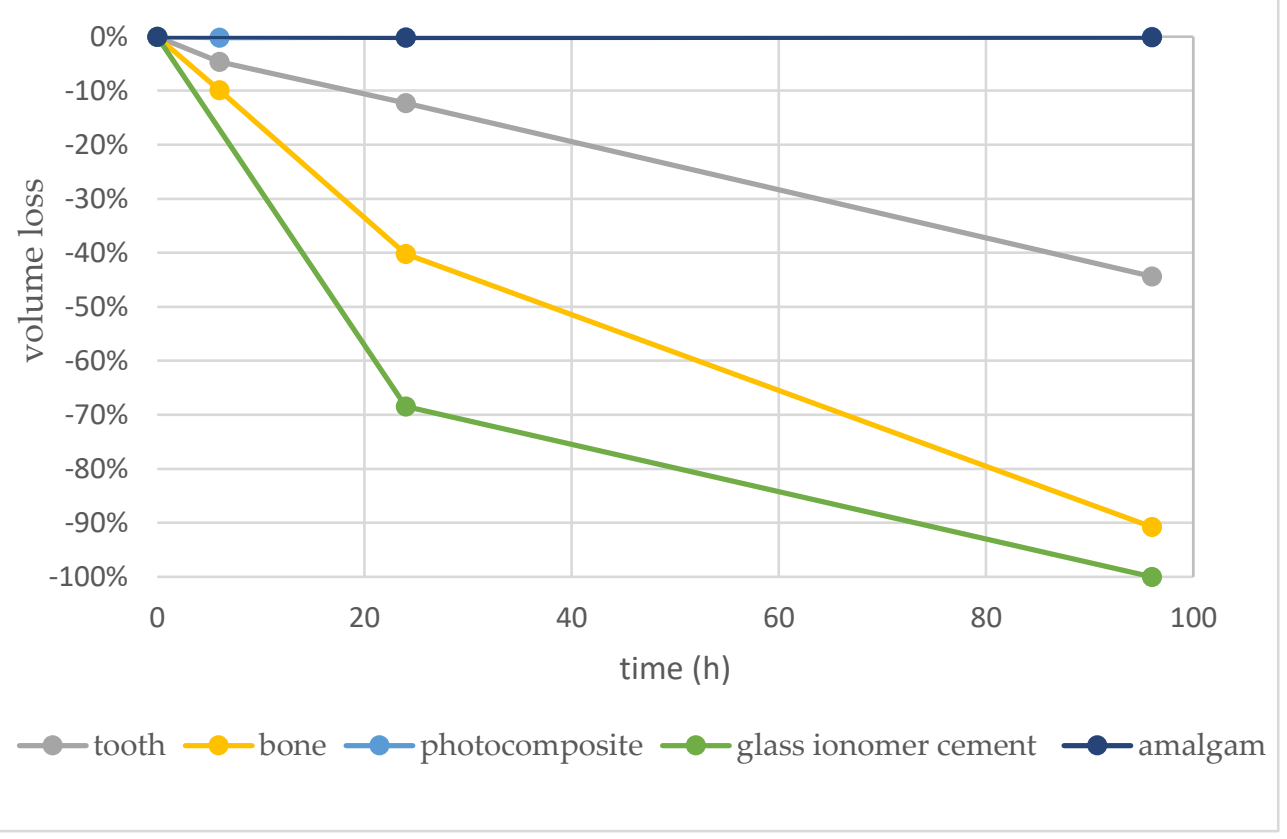

Figure 9. Graph representing volume loss over time in tooth, bone, composite, glass ionomer cement, and amalgam. The representation of photo-composite resin is hidden behind amalgam.

Table 1. Volume loss of dental materials and bone after exposure to $75 \%$ sulfuric acid. 


\begin{tabular}{|c|c|c|c|c|}
\hline time (h) & $\begin{array}{c}\mathbf{0} \\
\text { volume } 0 \mathrm{~h}\left(\mathrm{~mm}^{3}\right) \\
\end{array}$ & $\begin{array}{c}6 \\
\text { volume } 6 \mathrm{~h}\left(\mathrm{~mm}^{3}\right) \\
\end{array}$ & $\begin{array}{c}24 \\
\text { volume } 24 \mathrm{~h}\left(\mathrm{~mm}^{3}\right) \\
\end{array}$ & $\begin{array}{c}96 \\
\text { volume } 96 \mathrm{~h}\left(\mathrm{~mm}^{3}\right)\end{array}$ \\
\hline \multicolumn{5}{|l|}{ sample 32} \\
\hline dentine & 190.29 & 185.1 & 170.5 & 110.95 \\
\hline percentual loss & $0.0 \%$ & $-2.7 \%$ & $-10.4 \%$ & $-41.7 \%$ \\
\hline enamel & 12.81 & 8.56 & 7.64 & 2.03 \\
\hline percentual loss & $0.0 \%$ & $-33.2 \%$ & $-40.4 \%$ & $-84.2 \%$ \\
\hline tooth & 203.1 & 193.7 & 178.12 & 112.98 \\
\hline percentual loss & $0.0 \%$ & $-4.6 \%$ & $-12.3 \%$ & $-44.4 \%$ \\
\hline bone & 668.1 & 601.85 & 399.56 & 61.77 \\
\hline percentual loss & $0.0 \%$ & $-9.9 \%$ & $-40.2 \%$ & $-90.8 \%$ \\
\hline \multirow[t]{2}{*}{ sample 33} & 324.45 & 299.16 & 290.64 & 270.57 \\
\hline & $0.0 \%$ & $-7.8 \%$ & $-10.4 \%$ & $-16.6 \%$ \\
\hline \multirow[t]{2}{*}{ Photo-composite } & 16.46 & 16.43 & 16.45 & 16.46 \\
\hline & $0.0 \%$ & $-0.2 \%$ & $-0.1 \%$ & $0.0 \%$ \\
\hline \multicolumn{5}{|l|}{ sample 43} \\
\hline \multirow[t]{2}{*}{ glass ionomer } & 11.57 & 1 & 3.65 & 0 \\
\hline & $0.0 \%$ & 1 & $-68.5 \%$ & $-100.0 \%$ \\
\hline \multicolumn{5}{|l|}{ sample 47} \\
\hline amalgam & 33.42 & 33.43 & 33.37 & 33.4 \\
\hline & $0.0 \%$ & $0.0 \%$ & $-0.1 \%$ & $-0.1 \%$ \\
\hline
\end{tabular}

${ }^{1}$ No values for glass ionomer are a result of drop-out of the tooth filling during washing or drying process and skipping a round of Micro-CT scanning. However later to be found in the plastic sieves used in the process of washing.

\subsection{Digital matching and AI implementation in CBCT segmentation}

Digital matching of 3D reconstructed dental fillings, segmented from micro-CT, that survived acid bath, was successfully performed on all 4 localizations (2x amalgam, 2x composite). Restorations with glass ionomer cement dissolved and was not reproducible. One of the exemplary matchings over digital OPG is visualized on (Figure 10).

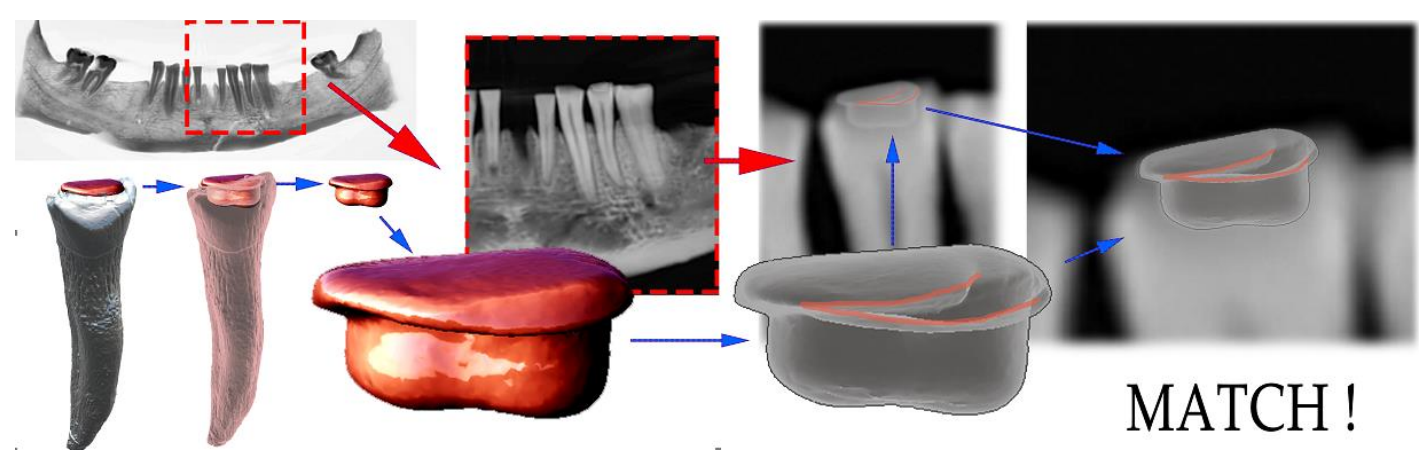

Figure 10. Reconstructed dental pattern of restoration from tooth 43 matched to $2 \mathrm{D}$ representing volume loss over time in tooth, bone, composite, glass ionomer cement, and amalgam. The representation of photo-composite resin is hidden behind amalgam.

Optional objective to compare manual (software) and AI segmentation methods of CBCT was fulfilled. This is possibly first implementation of advanced Artificial 
Intelligence algorithm utilization in clinical forensic research. The result of successful AI automatic segmentation took less than 5 minutes and provided similar results to manual segmentation which took more than 2 hours to a human operator. Small errors were observed in nerve canal segmentation and distal part of terminal molar contours (Figure 5b).

\subsection{ATR FTIR spectroscopy}

ATR FTIR spectroscopy was used to evaluate the chemical changes of the bone due to its degradation by $75 \%$ sulfuric acid. The spectrum of the original sample in (Figure 11a) contains characteristic bands of both organic and inorganic bone components. Amide I band at $1635 \mathrm{~cm}^{-1}$ and amide II band at $1540 \mathrm{~cm}^{-1}$, as well as week bands at 3080 $\mathrm{cm}^{-1}$ (Amide B) and $1240 \mathrm{~cm}^{-1}$ are characteristic of the collagen, which is a major organic component of bones [71]. The absorption band at $1020 \mathrm{~cm}^{-1}$ belongs to the phosphate group of hydroxyapatites (bioapatite, $\left.\mathrm{Ca}_{5}\left(\mathrm{PO}_{4}\right)_{3} \mathrm{OH}\right)$, the main inorganic component of bone, the bands at 1460, 1418 and $874 \mathrm{~cm}^{-1}$ belong to the carbonate group in $\mathrm{CaCO}_{3}$ [72].

After immersion of the bone in $75 \%$ sulfuric acid, the bands drop already after two hours, mainly in the amide region, which indicates a preferential removal of collagen from the bone surface. Significant bands are formed at 1100 and $1150 \mathrm{~cm}^{-1}$ (typical of the sulfate group in the calcium sulfate), which indicates the formation of a layer of calcium sulfate on the surface of the samples. Since the beam penetration depth at our ATR FTIR setting is $1-4 \mathrm{~mm}\left(1 \mathrm{~mm}\right.$ at $4000 \mathrm{~cm}^{-1}$ and $4 \mathrm{~mm}$ at $\left.600 \mathrm{~cm}^{-1}\right)$, we measure only a thin surface layer and the dominant bands belong to calcium sulphate forming on the surface after a short time. This complicates the process of evaluating changes in the material and limits us to measuring surfaces. Measurement of ATR FTIR after more than 24 hours failed to perform due to mechanical disruption of degraded bones due to pressure. The accumulation of calcium compounds on the bone surface was also confirmed by X-ray fluorescence (XRF) measurements, where after a short time there was an increase in the calcium content compared to phosphorus. XRF is a non-destructive analytical technique used to determine the elemental composition of materials. XRF analyzers determine the chemistry of a sample by measuring the fluorescent (or secondary) X-ray emitted from a sample when it is excited by a primary $\mathrm{X}$-ray source.

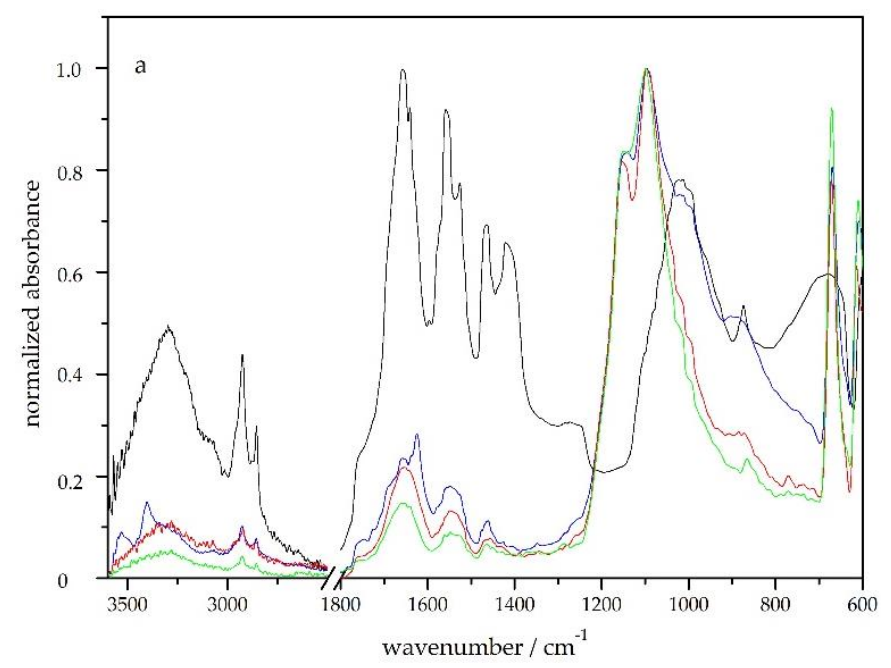

(a)

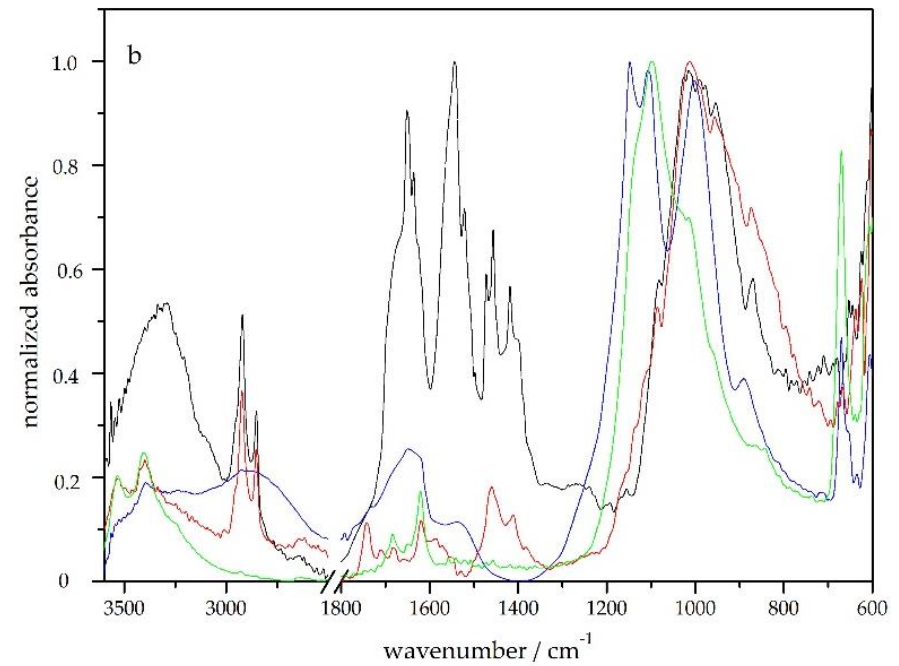

(b)

Figure 11. ATR FTIR spectra of bone (a) and tooth (b) immersed in $75 \%$ sulfuric acid for $2 \mathrm{~h}$ (blue line), $6 \mathrm{~h}$ (red line), $24 \mathrm{~h}$ (green line) and original sample (black line).

With the same measurements on the model tooth (Figure 11 b), teeth showed as more stable and were naturally expected to last longer than bone [11]. The main absorption bands of the tooth are located in the same areas as the bone. The band at $1020 \mathrm{~cm}^{-1}$ was retained after six and partly after 24 hours in sulfuric acid, while the absorption bands 
belonging to collagen (1641 and $1540 \mathrm{~cm}^{-1}$ ) decreased significantly, thus leading to a faster loss of organic components. In the spectra measured at different times of acid action, there is not only a simple decrease in bands, but the differences are larger (e.g., between two and six hours of exposure to $75 \%$ sulfuric acid), which is related to the fact that the tooth is a multilayer material and after dissolving the enamel we can observe dentin (the spectrum after $6 \mathrm{~h}$ of sulfuric acid treatment corresponds to the spectrum of dentin, where bands between 1410 and $1650 \mathrm{~cm}^{-1}$ are typical for carbonate overlapped with collagen) [60]. As in the case of bone, $\mathrm{CaSO}_{4}$ formation was observed also on the tooth surface in all of the samples.

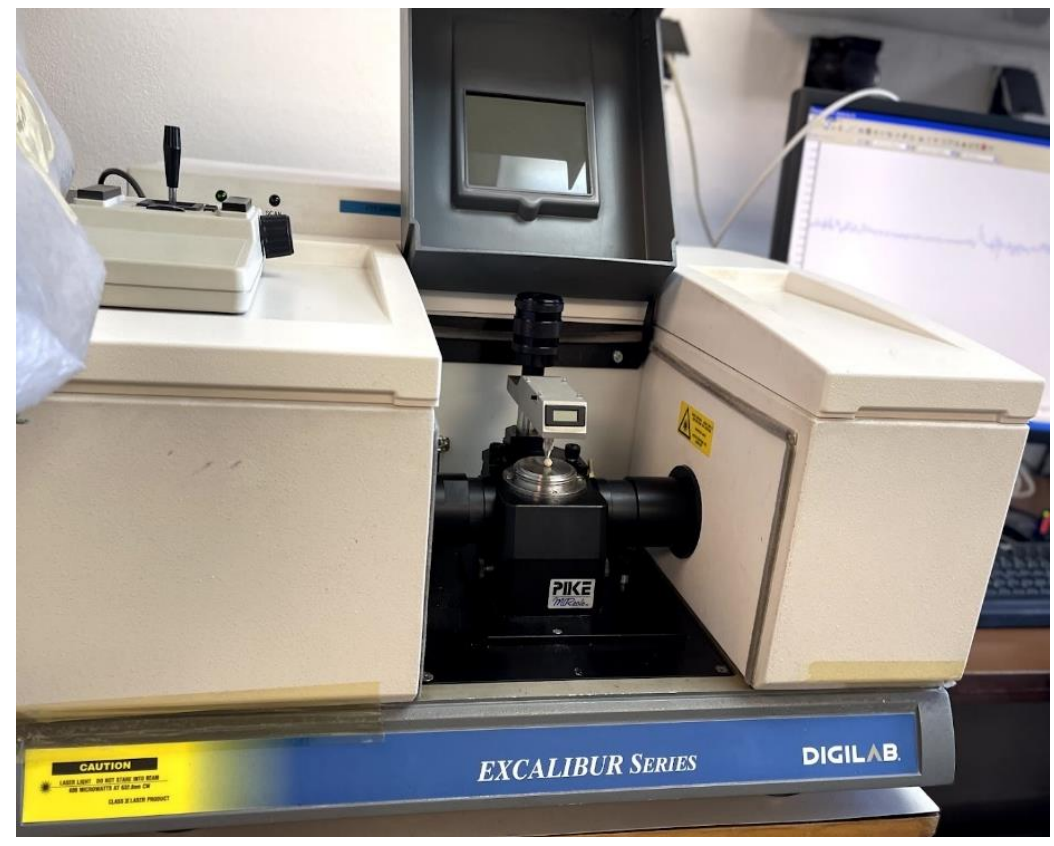

(a)

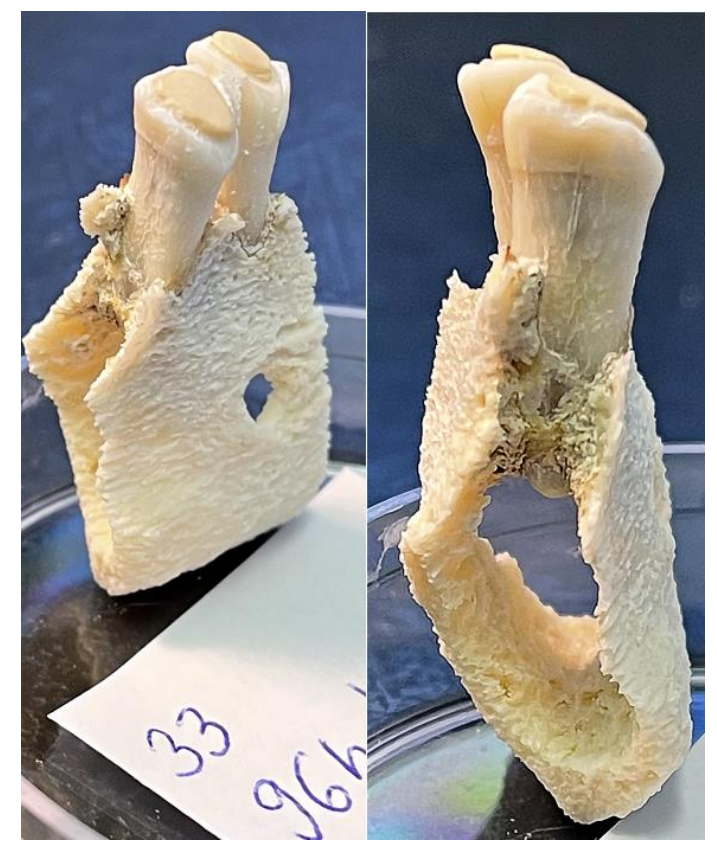

(b)

Figure 12. FTIR spectrophotometer Varian Excalibur Digilab FTS 3000MX, (Palo Alto, CA, USA), equipped with the ATR adapter with diamond crystal (a) sample placed in the spectrophotometer; (b) example of fragile sample 33 after $96 \mathrm{~h}$ of acid bath exposure - not suitable for sensor pressure. 


\section{Discussion}

Results showed outstanding resistance of high copper non-gamma 2 amalgam and photo-composite resin to impact of $75 \%$ sulfuric acid. Even after 96 hours in the $75 \%$ sulfuric acid bath the volumetric loss was less approximately $0.1 \%$ in amalgam and none in photo-composite (Table 1 and Figure 9). These findings correlate to complex research presented in the master thesis of Trapp 2018 from School of Medicine Boston University $[73,74]$ that provides comprehensive research of the effects of household corrosive substances on teeth restorations. Particularly the author provides an evaluation of effect of solution of 93.2\% sulfuric acid on silver amalgam (Figure 4.3, page 62 [73]). The silver amalgam tooth filling had minimal changes and was as well resistant to sulfuric acid even after 264 hour-exposure. To approximate these findings, it is important to understand difference between high silver and high copper amalgam (Table 2). In general, the high copper amalgam is stronger and it might be expected even more resilient to sulfuric acid solutions than high silver amalgam.

Table 2. Comparison of high silver and high copper amalgam

\begin{tabular}{cc}
\hline high silver amalgam & high copper amalgam \\
ANA 70 | Nordiska Dental & ANA2000 | Nordiska Dental \\
\hline Silver shining & Long-lasting, resistant and strong \\
Dimensional change: & Dimensional change: \\
$24 \mathrm{~h}+0,1 \%$ & $24 \mathrm{~h}+0,02 \%$ \\
& \\
PHYSICAL PROPERTIES & PHYSICAL PROPERTIES \\
Compressive strength: & Compressive strength: \\
1h - $110 \mathrm{MPa}$ & $1 \mathrm{~h}-140 \mathrm{MPa}$ \\
$24 \mathrm{~h}-420 \mathrm{MPa}$ & $24 \mathrm{~h}-450 \mathrm{MPa}$ \\
& \\
ALLOY COMPOSITION & ALLOY COMPOSITION \\
Ag $69,9 \%$ & Ag $43,1 \%$ \\
Sn $18,8 \%$ & Sn $30,8 \%$ \\
Cu $10,2 \%$ & Cu $26,1 \%$ \\
Zn $1,1 \%$ & \\
\hline
\end{tabular}

However, this resistance to sulfuric acid was not shared with the third used dental material - glass-ionomer cement. The glass-ionomer preparation dissolved completely after 96 hours. Results suggest that the dental restorations from typical dental restorations as high copper non-gamma 2 amalgam and photo-composite methacrylate resins, have minimal volume loss and deformation under $75 \%$ sulfuric acid exposure. And might serve for dental pattern reconstruction during the process of identification.

The acid concentrations are very important for the final morphological and chemical impact. This is confirmed with research of Raj et al. in 2013 [23]. It evaluated the resistance of teeth to acids ( $37 \%$ conc. $\mathrm{HCl}), 65 \%$ conc. $\mathrm{HNO}_{3}$ ) and $96 \%$ conc. $\mathrm{H}_{2} \mathrm{SO}_{4}$ ). The research confirms that teeth will completely dissolve in $\mathrm{HCl}$ and $\mathrm{HNO}_{3}$, however in 8 hours, they still retain morphology in $\mathrm{H}_{2} \mathrm{SO}_{4}$. Concentration of the acid is one important aspect, the time of exposure another. Other studies [24] conducted earlier by Dr. Mazza and published in the Journal of Forensic Sciences were initiated by judges in so called "mafia" crimes to examine whether the body could be destroyed by immersion in acids and whether any remains could be identified at all. The aim of this study was to observe the behavior of teeth exposed to four types of acidic solutions. The teeth were placed in plastic jars with $25 \mathrm{ml}$ of acid and observed. Experience has shown that the teeth are completely dissolved after 14 hours of immersion in a 37\% hydrochloric acid solution, while after 90 hours in $96 \%$ sulfuric acid the destruction of the samples is still incomplete. In nitric acid, 
the teeth completely dissolved in 12 hours and in 17 hours in solution of hydrochloric acid / nitric acid 1:3, it was possible to recognize the characteristic morphological features of dental tissues and structures up to advanced stages of degradation.

In regards to significant differences of acid impact depending on its concentration, a group of forensic scientists decided to engage in research to dispel myths and test whether the body, after soaking in acid, actually does or does not disintegrate within a few minutes. Massimo Grillo of the University of Palermo and his colleagues presented their results at a meeting of the American Academy of Forensic Sciences. When the researchers placed pieces of pig carcasses in sulfuric acid, it took several days for the meat to dissolve. When water was added to the mixture, they were able to reduce the dissolution time to 12 hours for muscle and cartilage and two days for bone. This is a valuable observation. It is known that acid should be poured into water and not the other way around, in the case of sulfuric acid it is a strong exothermic reaction producing a lot of heat and fumes of explosive gases. Dutch research [75] confirms the experience of forensic experts that, in addition to macroscopic findings such as bone residues and artificial teeth, in both cases clear microscopic bone residues were found: (partially) digested bone, thin-walled structures and recrystallized calcium phosphate. Although some believe that the body can be completely dissolved in acid, at least some of these microscopic residues are always found. These microscopic residues might be future path for implementation of artificial intelligence algorithms in modern reconstruction of dental patterns.

As a limitation of this study might be considered that research has been performed on old - dried out skeleton. This is not representative in considerations for reproduction of processes accompanying complete human tissues exposed to acids. Another limitation is on the contrary the fact that dental restorations were only a week old before exposure to acid bath and were never exposed to native environment of the oral cavity. Both of these aspects might not be relevant for presented results, however, shall be taken into consideration before approximation of these results to different environments and situations.

\section{Conclusions}

The primary working hypothesis was confirmed and proved that typical dental restorations from high copper non-gamma 2 amalgam and photo-composite nano hybrid methacrylate modified polysiloxane (organically modified ceramic) dimethacrylate resins, have minimal volume loss and deformation under $75 \%$ sulfuric acid exposure.

The secondary hypothesis was also confirmed premising that 3D reconstructed dental patterns can be utilized for matching with 2D dental records represented with intraoral digital photo and OPG scan.

CBCT, Micro-CT and ATR-FTIR spectroscopy represent a suitable combination of technologies for successful morphological and spectroscopic reconstruction of dental patterns.

Advanced artificial intelligence algorithm was successfully implemented to perform automatic segmentation of the CBCT scan with separate STL outputs of mandibular bone, teeth and nerve canal.

Supplementary Materials: The following supporting information can be downloaded at: Data supporting reported results are freely available at: https://drive.google.com/drive/folders/1UqhTbdDWZwxHamUMh6xxgqx3UDC1wimF?usp=sharing The online location provides full digital photo set and STL segmentations of every from 4-stages of degradation for bone and separately for teeth in that particular segment. Also, digital iTero scan of the mandible and CT segmented with $\mathrm{AI}$ is provided in this location.

Funding: This research was funded by the KEGA grant agency of the Ministry of Education, Science, Research, and Sport of the Slovak Republic (Grant No. 081UK-4/2021) as well with COST Association registered as legal Entity Brussels: 0829090573, supported by the EU framework Programme Horizon 2020, MVTS COST action CA16101 Multi-modal Imaging of Forensic Science Evidence (MULTI-FORESEE). 
Institutional Review Board Statement: The study was conducted according to the guidelines of the Declaration of Helsinki, and no approval was necessary by the Ethics Committee. Ethical review and approval were waived for this study, as the study did not involve any living animals or humans.

Informed Consent Statement: Patient consent was waived due to the fact no patients were involved in this study.

Data Availability Statement: We fully adhere to Data Availability Statements in section "MDPI Research Data Policies" at https://www.mdpi.com/ethics.

Data supporting reported results are freely available at: https://drive.google.com/drive/folders/1UqhTbdDWZwxHamUMh6xxgqx3UDC1wimF?usp=sharing The online location provides full digital photo set and STL segmentations of every from 4-stages of degradation for bone and separately for teeth in that particular segment. Also, digital iTero scan of the mandible and CT segmented with $\mathrm{AI}$ is provided in this location.

Acknowledgments: We acknowledge support and digital dental lab infrastructure of 3Dent Medical s.r.o company as well as dental clinic Sangre Azul s.r.o.

Conflicts of Interest: The authors declare no conflict of interest.

\section{Appendix A}

This optional section contains details and data supplemental to the main text of the article, particularly interesting digital visualization of sample micro-CT segmentations (Figure A1) and digital photo-documentation of the samples after acid-bath (Figure A2). These experimental details remain very useful to understanding and reproducing the research presented above; STLS are provided as Supplementary data publicly available online. 

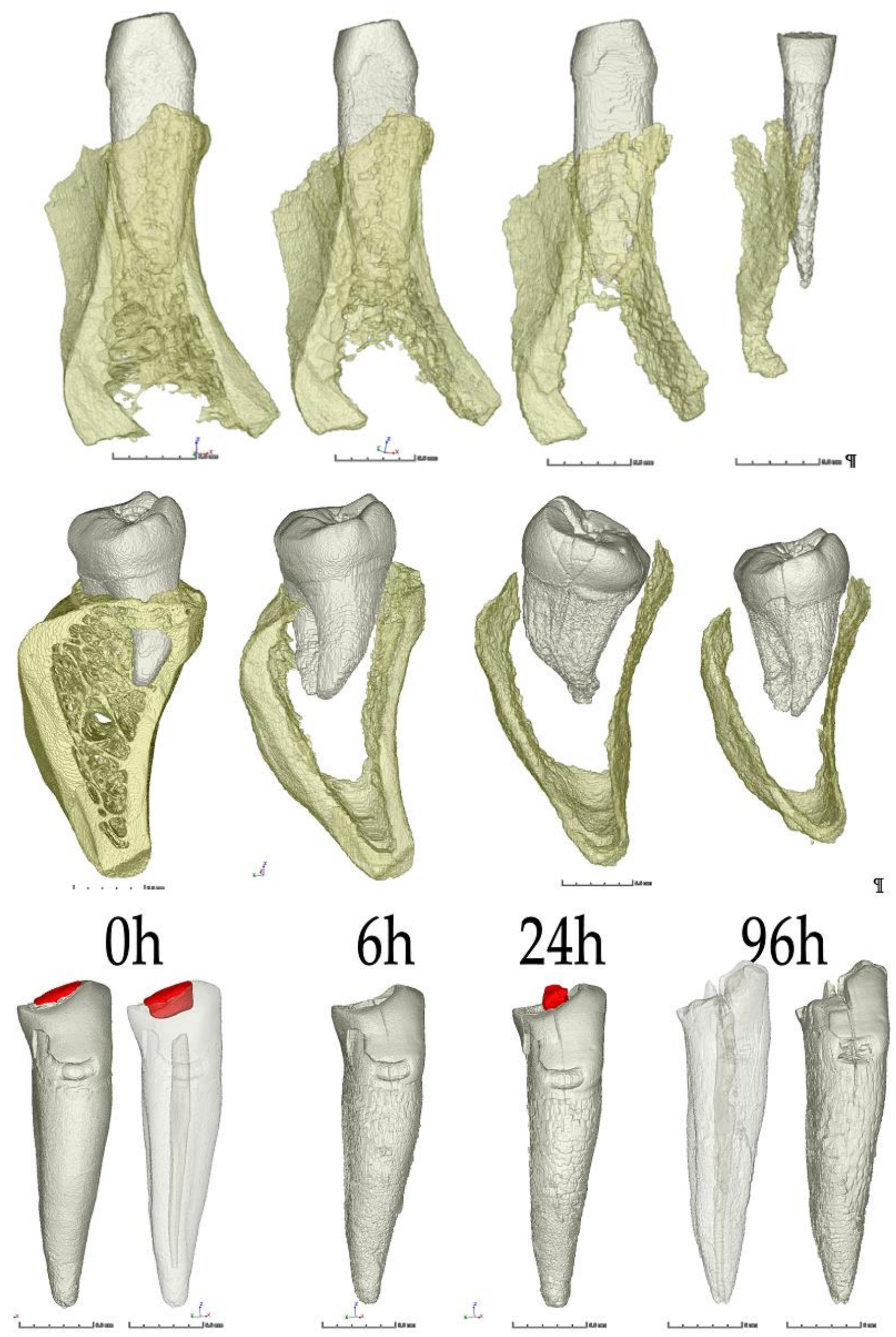


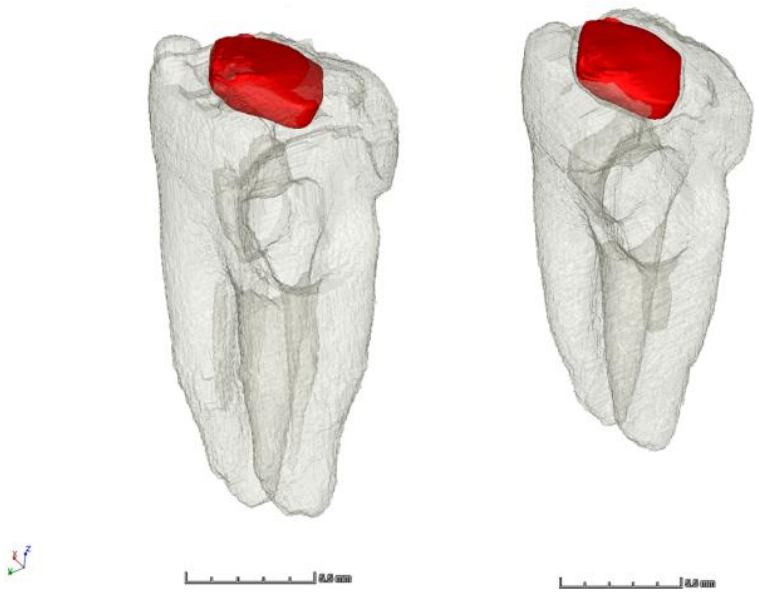

Figure A1. Digital visualization of sample micro-CT segmentations. From top row: (1 - the first row) Sample 32 intact tooth; (2) Sample 38 with untreated caries; (3) Sample 43 with glass ionomer cement; (4 - the last row) Sample 47 with amalgam

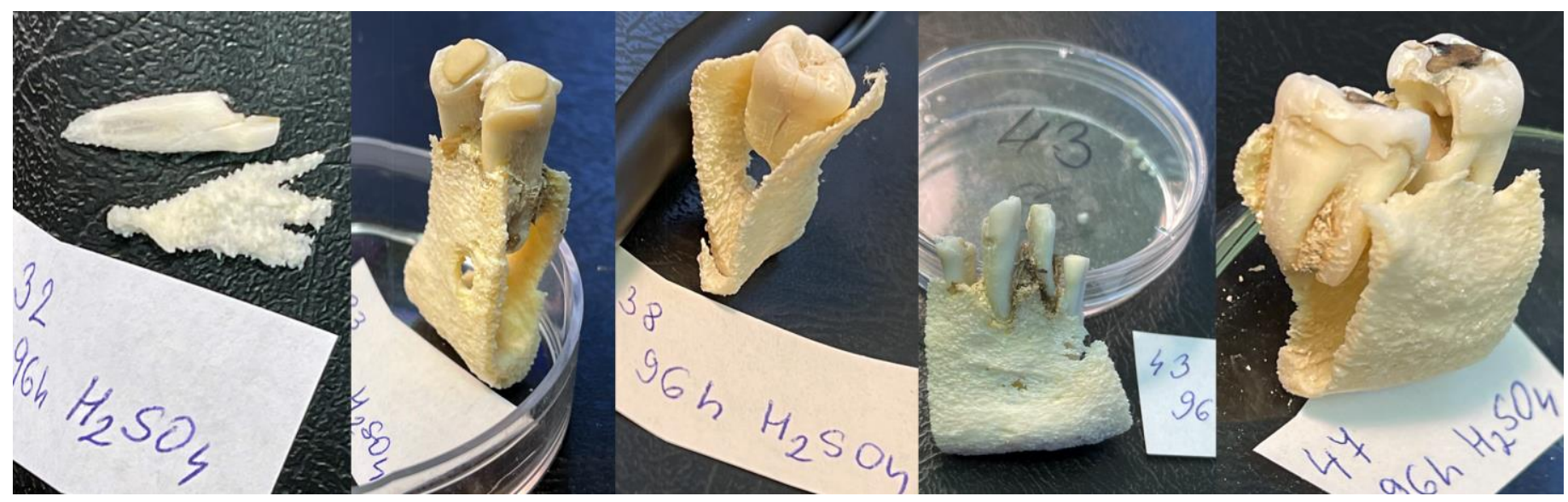

Figure A2. Photo-documentation of the samples after 96 hours in $75 \%$ sulfuric acid bath.

\section{References}

1. Martin-de-las-Heras, S.; Valenzuela, A.; Luna, J. de D.; Bravo, M. The Utility of Dental Patterns in Forensic Dentistry. Forensic science international 2010, 195, 166.e1-166.e5, doi:10.1016/J.FORSCIINT.2009.11.004.

2. Ata-Ali, J.; Ata-Ali, F. Forensic Dentistry in Human Identification: A Review of the Literature. Journal of clinical and experimental dentistry 2014, 6, doi:10.4317/JCED.51387.

3. Latham, K.E.; Bartelink, E.J.; Finnegan, M. New Perspectives in Forensic Human Skeletal Identification. New Perspectives in Forensic Human Skeletal Identification 2017, 1-356.

4. Izham, A.; Auerkari, E.I. The Use of Radiology CBCT in Odontology Forensic. AIP Conference Proceedings 2021, 2344, 050012, doi:10.1063/5.0047278.

5. Rutty, G.N.; Brough, A.; Biggs, M.J.P.; Robinson, C.; Lawes, S.D.A.; Hainsworth, S. v. The Role of MicroComputed Tomography in Forensic Investigations. Forensic Science International 2013, 225, 60-66, doi:10.1016/J.FORSCIINT.2012.10.030.

6. Gaêta-Araujo, H.; Nascimento, E.H.L.; Brasil, D.M.; Madlum, D.V.; Haiter-Neto, F.; Oliveira-Santos, C. Influence of Reconstruction Parameters of Micro-Computed Tomography on the Analysis of Bone Mineral Density. Imaging Science in Dentistry 2020, 50, 153, doi:10.5624/ISD.2020.50.2.153. 
7. Thurzo, A.; Kosnáčová, H.S.; Kurilová, V.; Kosmel', S.; Beňuš, R.; Moravanský, N.; Kováč, P.; Kuracinová, K.M.; Palkovič, M.; Varga, I. Use of Advanced Artificial Intelligence in Forensic Medicine, Forensic Anthropology and Clinical Anatomy. Healthcare 2021, Vol. 9, Page 1545 2021, 9, 1545, doi:10.3390/HEALTHCARE9111545.

8. Ezhov, M.; Gusarev, M.; Golitsyna, M.; Yates, J.M.; Kushnerev, E.; Tamimi, D.; Aksoy, S.; Shumilov, E.; Sanders, A.; Orhan, K. Clinically Applicable Artificial Intelligence System for Dental Diagnosis with CBCT. Scientific Reports 2021 11:1 2021, 11, 1-16, doi:10.1038/s41598-021-94093-9.

9. Mudrak, J. Artificial Intelligence and Deep Learning in Dental Radiology Available online: https://www.oralhealthgroup.com/features/artificial-intelligence-and-deep-learning-in-dental-radiology-away-forward-in-point-of-care-radiology/ (accessed on 28 December 2021).

10. Lebon, M.; Zazzo, A.; Reiche, I. Screening in Situ Bone and Teeth Preservation by ATR-FTIR Mapping. Palaeogeography, Palaeoclimatology, Palaeoecology 2014, 416, 110-119, doi:10.1016/J.PALAEO.2014.08.001.

11. France, C.A.M.; Sugiyama, N.; Aguayo, E. Establishing a Preservation Index for Bone, Dentin, and Enamel Bioapatite Mineral Using ATR-FTIR. Journal of Archaeological Science: Reports 2020, 33, doi:10.1016/j.jasrep.2020.102551.

12. Schrader, B.A. History and Scope of Forensic Odontology. Forensic Odontology: Principles and Practice 2018, 1923, doi:10.1016/B978-0-12-805198-6.00002-5.

13. Clement, J.G. Odontology. Encyclopedia of Forensic Sciences: Second Edition 2013, 106-113, doi:10.1016/B978-0-12382165-2.00018-0.

14. Putrino, A.; Putrino, A.; Raso, M.; Bruti, V.; Marinelli, E.; Barbato, E.; Galluccio, G. Forensic Dentistry in Human Identification: Overview of a Modern Science. Transylvanian Review 2021, 29.

15. Belcastro, M.G.; Mariotti, V.; Riga, A.; Bonfiglioli, B.; Frayer, D.W. Tooth Fractures in the Krapina Neandertals. Journal of human evolution 2018, 123, 96-108, doi:10.1016/J.JHEVOL.2018.06.009.

16. Milella, M.; Belcastro, M.G.; Mariotti, V.; Nikita, E. Estimation of Adult Age-at-Death from Entheseal Robusticity: A Test Using an Identified Italian Skeletal Collection. American journal of physical anthropology 2020, 173, 190-199, doi:10.1002/AJPA.24083.

17. Cameriere, R.; de Luca, S.; Egidi, N.; Bacaloni, M.; Maponi, P.; Ferrante, L.; Cingolani, M. Automatic Age Estimation in Adults by Analysis of Canine Pulp/Tooth Ratio: Preliminary Results. Journal of Forensic Radiology and Imaging 2015, 3, 61-66, doi:10.1016/J.JOFRI.2014.10.001.

18. Belcastro, M.G.; Nicolosi, T.; Sorrentino, R.; Mariotti, V.; Pietrobelli, A.; Bettuzzi, M.; Morigi, M.P.; Benazzi, S.; Talamo, S.; Miari, M.; et al. Unveiling an Odd Fate after Death: The Isolated Eneolithic Cranium Discovered in the Marcel Loubens Cave (Bologna, Northern Italy). PloS one 2021, 16, doi:10.1371/JOURNAL.PONE.0247306.

19. Rui Filipe Libânio Osório Marta Strategies for the Identification of the Missing: A Review of the Contributions of Forensic Anthropology and Forensic Dentistry; 2021;

20. Bewes, J.; Low, A.; Morphett, A.; Pate, F.D.; Henneberg, M. Artificial Intelligence for Sex Determination of Skeletal Remains: Application of a Deep Learning Artificial Neural Network to Human Skulls. Journal of Forensic and Legal Medicine 2019, 62, 40-43, doi:10.1016/J.JFLM.2019.01.004.

21. Kadashetti, V.; Shivakumar, K.; Baad, R.; Vibhute, N.; Belgaumi, U.; Bommanavar, S.; Kamate, W. Effect of Concentrated Acids on Teeth: A Forensic Approach; An In-Vitro Study. Journal of Datta Meghe Institute of Medical Sciences University 2021, 16, 283, doi:10.4103/JDMIMSU.JDMIMSU_32_18.

22. Guendalina, G.; Stefano, T.; Salvatore, A.; Paolo, B.; Giorgia, B.; Ilaria, G.; Riccardo, Z. Analysis of the Corrosive Effects of Hydrochloric Acid $(\mathrm{HCl})$ on Human Bone: Preliminary Microscopic Study and Observations for Forensic Purposes. Forensic Science International 2021, 329, 111095, doi:10.1016/J.FORSCIINT.2021.111095.

23. Raj, M.; Boaz, K.; Srikant, N. Are Teeth Evidence in Acid Environment. Journal of Forensic Dental Sciences 2013, 5, 7, doi:10.4103/0975-1475.114536. 
24. Mazza, A.; Merlati, G.; Savio, C.; Fassina, G.; Menghini, P.; Danesino, P. Observations on Dental Structures When Placed in Contact with Acids: Experimental Studies to Aid Identification Processes; 2005; Vol. 50;.

25. Krishan, K.; Kanchan, T.; Garg, A.K. Dental Evidence in Forensic Identification - An Overview, Methodology and Present Status. The Open Dentistry Journal 2015, 9, 250, doi:10.2174/1874210601509010250.

26. Sarment, D.P.; Christensen, A.M. The Use of Cone Beam Computed Tomography in Forensic Radiology. Journal of Forensic Radiology and Imaging 2014, 2, 173-181, doi:10.1016/J.JOFRI.2014.09.002.

27. Trochesset, D.A.; Serchuk, R.B.; Colosi, D.C. Generation of Intra-Oral-like Images from Cone Beam Computed Tomography Volumes for Dental Forensic Image Comparison. Journal of Forensic Sciences 2014, 59, 510-513, doi:10.1111/1556-4029.12336.

28. Lucena, J.; Mora, E.; Rodriguez, L.; Muñoz, M.; Cantin, M.G.; Fonseca, G.M. Cone Beam Computed Tomography (CBCT) as a Tool for the Analysis of Nonhuman Skeletal Remains in a Medico-Legal Setting. Forensic Science International 2016, 266, e32-e37, doi:10.1016/J.FORSCIINT.2016.06.025.

29. Matsuda, S.; Yoshida, H.; Ebata, K.; Shimada, I.; Yoshimura, H. Forensic Odontology with Digital Technologies: A Systematic Review. Journal of Forensic and Legal Medicine 2020, 74, 102004, doi:10.1016/J.JFLM.2020.102004.

30. Viner, M.D.; Robson, J. Post-Mortem Forensic Dental Radiography - a Review of Current Techniques and Future Developments. Journal of Forensic Radiology and Imaging 2017, 8, 22-37, doi:10.1016/J.JOFRI.2017.03.007.

31. Dalessandri, D.; Tonni, I.; Laffranchi, L.; Migliorati, M.; Isola, G.; Visconti, L.; Bonetti, S.; Paganelli, C. 2D vs. 3D Radiological Methods for Dental Age Determination around 18 Years: A Systematic Review. Applied Sciences 2020, Vol. 10, Page 3094 2020, 10, 3094, doi:10.3390/APP10093094.

32. Aktuna Belgın, C.; Serindere, G.; Orhan, K. Accuracy and Reliability of Enamel and Dentin Thickness Measurements on Micro-Computed Tomography and Digital Periapical Radiographs\#. Journal of Forensic Radiology and Imaging 2019, 18, 32-36, doi:10.1016/J.JOFRI.2019.05.006.

33. Ge, Z. pu; Yang, P.; Li, G.; Zhang, J. zong; Ma, X. chen Age Estimation Based on Pulp Cavity/Chamber Volume of 13 Types of Tooth from Cone Beam Computed Tomography Images. International Journal of Legal Medicine 2016, 130, 1159-1167, doi:10.1007/S00414-016-1384-6/TABLES/5.

34. Norman, D.G.; Baier, W.; Watson, D.G.; Burnett, B.; Painter, M.; Williams, M.A. Micro-CT for Saw Mark Analysis on Human Bone. Forensic Science International 2018, 293, 91-100, doi:10.1016/J.FORSCIINT.2018.10.027.

35. Bouletreau, P.; Makaremi, M.; Ibrahim, B.; Louvrier, A.; Sigaux, N. Artificial Intelligence: Applications in Orthognathic Surgery. Journal of Stomatology, Oral and Maxillofacial Surgery 2019, 120, 347-354, doi:10.1016/J.JORMAS.2019.06.001.

36. Bewes, J.; Low, A.; Morphett, A.; Pate, F.D.; Henneberg, M. Artificial Intelligence for Sex Determination of Skeletal Remains: Application of a Deep Learning Artificial Neural Network to Human Skulls. Journal of Forensic and Legal Medicine 2019, 62, 40-43, doi:10.1016/J.JFLM.2019.01.004.

37. Bewes, J.; Low, A.; Morphett, A.; Pate, F.D.; Henneberg, M. Artificial Intelligence for Sex Determination of Skeletal Remains: Application of a Deep Learning Artificial Neural Network to Human Skulls. Journal of Forensic and Legal Medicine 2019, 62, 40-43, doi:10.1016/J.JFLM.2019.01.004.

38. Himanshi Narang Application of Artificial Intelligence in the Field of Forensic Medicine. Indian Journal of Forensic Medicine E Toxicology 2021, 15, doi:10.37506/IJFMT.V15I4.

39. Rijn, R.R. van; Luca, A. de Three Reasons Why Artificial Intelligence Might Be the Radiologist's Best Friend. https://doi.org/10.1148/radiol.2020200855 2020, 296, 159-160, doi:10.1148/RADIOL.2020200855.

40. Khanagar, S.B.; Vishwanathaiah, S.; Naik, S.; A. Al-Kheraif, A.; Devang Divakar, D.; Sarode, S.C.; Bhandi, S.; Patil, S. Application and Performance of Artificial Intelligence Technology in Forensic Odontology - A Systematic Review. Legal Medicine 2021, 48, 101826, doi:10.1016/J.LEGALMED.2020.101826. 
41. Makaremi, M.; Lacaule, C.; Mohammad-Djafari, A. Deep Learning and Artificial Intelligence for the Determination of the Cervical Vertebra Maturation Degree from Lateral Radiography. Entropy 2019, Vol. 21, Page 1222 2019, 21, 1222, doi:10.3390/E21121222.

42. Almalki, Y.E.; Qayyum, A.; Irfan, M.; Haider, N.; Glowacz, A.; Alshehri, F.M.; Alduraibi, S.K.; Alshamrani, K.; Basha, M.A.A.; Alduraibi, A.; et al. A Novel Method for COVID-19 Diagnosis Using Artificial Intelligence in Chest X-Ray Images. Healthcare 2021, Vol. 9, Page 522 2021, 9, 522, doi:10.3390/HEALTHCARE9050522.

43. Ren, K.; Li, N.; Liang, X.; Wang, L.; Li, J.; Sun, J. Investigating the New Orientation of Wound Age Estimation in Forensic Medicine Based on Biological Omics Data Combined with Artificial Intelligence Algorithms. PervasiveHealth: Pervasive Computing Technologies for Healthcare 2020, 54-59, doi:10.1145/3433996.3434007.

44. Li, L.; Qin, L.; Xu, Z.; Yin, Y.; Wang, X.; Kong, B.; Bai, J.; Lu, Y.; Fang, Z.; Song, Q.; et al. Using Artificial Intelligence to Detect COVID-19 and

Community-Acquired Pneumonia Based on Pulmonary CT:

Evaluation of the Diagnostic Accuracy. https://doi.org/10.1148/radiol.2020200905 2020, 296, E65E71, doi:10.1148/RADIOL.2020200905.

45. Thurzo, A.; Kurilová, V.; Varga, I. Artificial Intelligence in Orthodontic Smart Application for Treatment Coaching and Its Impact on Clinical Performance of Patients Monitored with AI-TeleHealth System. Healthcare 2021, Vol. 9, Page 1695 2021, 9, 1695, doi:10.3390/HEALTHCARE9121695.

46. Punyani, P.; Gupta, R.; Kumar, A. Neural Networks for Facial Age Estimation: A Survey on Recent Advances. Artificial Intelligence Review 2019 53:5 2019, 53, 3299-3347, doi:10.1007/S10462-019-09765-W.

47. Miki, Y.; Muramatsu, C.; Hayashi, T.; Zhou, X.; Hara, T.; Katsumata, A.; Fujita, H. Classification of Teeth in ConeBeam CT Using Deep Convolutional Neural Network. Computers in Biology and Medicine 2017, 80, 24-29, doi:10.1016/J.COMPBIOMED.2016.11.003.

48. Bernauer, S.A.; Zitzmann, N.U.; Joda, T. The Use and Performance of Artificial Intelligence in Prosthodontics: A Systematic Review. Sensors 2021, Vol. 21, Page 6628 2021, 21, 6628, doi:10.3390/S21196628.

49. Müller, A.; Mertens, S.M.; Göstemeyer, G.; Krois, J.; Schwendicke, F. Barriers and Enablers for Artificial Intelligence in Dental Diagnostics: A Qualitative Study. Journal of Clinical Medicine 2021, Vol. 10, Page 1612 2021, 10, 1612, doi:10.3390/JCM10081612.

50. Bayrakdar, S.K.; Orhan, K.; Bayrakdar, I.S.; Bilgir, E.; Ezhov, M.; Gusarev, M.; Shumilov, E. A Deep Learning Approach for Dental Implant Planning in Cone-Beam Computed Tomography Images. BMC Medical Imaging 2021, 21, 1-9, doi:10.1186/S12880-021-00618-Z/TABLES/3.

51. Orhan, K.; Bilgir, E.; Bayrakdar, I.S.; Ezhov, M.; Gusarev, M.; Shumilov, E. Evaluation of Artificial Intelligence for Detecting Impacted Third Molars on Cone-Beam Computed Tomography Scans. Journal of Stomatology, Oral and Maxillofacial Surgery 2021, 122, 333-337, doi:10.1016/J.JORMAS.2020.12.006.

52. Orhan, K.; Bayrakdar, I.S.; Ezhov, M.; Kravtsov, A.; Özyürek, T. Evaluation of Artificial Intelligence for Detecting Periapical Pathosis on Cone-Beam Computed Tomography Scans. International Endodontic Journal 2020, 53, 680689, doi:10.1111/IEJ.13265.

53. Silva, T.P.; Hughes, M.M.; Menezes, L. dos S.; Melo, M. de F.B. de; Takeshita, W.M.; Freitas, P.H.L. de Artificial Intelligence-Based Cephalometric Landmark Annotation and Measurements According to Arnett's Analysis: Can We Trust a Bot to Do That? https://doi.org/10.1259/dmfr.20200548 2021, 20200548, doi:10.1259/DMFR.20200548.

54. Hamet, P.; Tremblay, J. Artificial Intelligence in Medicine. Metabolism 2017, 69, S36-S40, doi:10.1016/J.METABOL.2017.01.011.

55. Hamet, P.; Tremblay, J. Artificial Intelligence in Medicine. Metabolism 2017, 69, S36-S40, doi:10.1016/J.METABOL.2017.01.011. 
56. Bewes, J.; Low, A.; Morphett, A.; Pate, F.D.; Henneberg, M. Artificial Intelligence for Sex Determination of Skeletal Remains: Application of a Deep Learning Artificial Neural Network to Human Skulls. Journal of Forensic and Legal Medicine 2019, 62, 40-43, doi:10.1016/J.JFLM.2019.01.004.

57. YT, F.; Q, L.; T, X.; YF, L.; SY, M.; BF, Z. New Opportunities and Challenges for Forensic Medicine in the Era of Artificial Intelligence Technology. Fa yi xue za zhi 2020, 36, 77-85, doi:10.12116/J.ISSN.1004-5619.2020.01.016.

58. Hung, K.; Montalvao, C.; Tanaka, R.; Kawai, T.; Bornstein, M.M. The Use and Performance of Artificial Intelligence Applications in Dental and Maxillofacial Radiology: A Systematic Review. https://doi.org/10.1259/dmfr.20190107 2019, 49, doi:10.1259/DMFR.20190107.

59. Tanikawa, C.; Yamashiro, T. Development of Novel Artificial Intelligence Systems to Predict Facial Morphology after Orthognathic Surgery and Orthodontic Treatment in Japanese Patients. Scientific Reports 2021 11:1 2021, 11, 1-11, doi:10.1038/s41598-021-95002-w.

60. Lopes, C. de C.A.; Limirio, P.H.J.O.; Novais, V.R.; Dechichi, P. Fourier Transform Infrared Spectroscopy (FTIR) Application Chemical Characterization of Enamel, Dentin and Bone. https://doi.org/10.1080/05704928.2018.1431923 2018, 53, 747-769, doi:10.1080/05704928.2018.1431923.

61. Wang, Q.; Zhang, Y.; Lin, H.; Zha, S.; Fang, R.; Wei, X.; Fan, S.; Wang, Z. Estimation of the Late Postmortem Interval Using FTIR Spectroscopy and Chemometrics in Human Skeletal Remains. Forensic Science International 2017, 281, 113-120, doi:10.1016/J.FORSCIINT.2017.10.033.

62. Chophi, R.; Sharma, S.; Singh, R. Forensic Analysis of Red Lipsticks Using ATR-FTIR Spectroscopy and Chemometrics. Forensic Chemistry 2020, 17, 100209, doi:10.1016/J.FORC.2019.100209.

63. Irfanita, N.; Jaswir, I.; Mirghani, M.E.S.; Sukmasari, S.; Ardini, Y.D.; Lestari, W. Rapid Detection of Gelatin in Dental Materials Using Attenuated Total Reflection Fourier Transform Infrared Spectroscopy (ATR-FTIR). Journal of Physics: Conference Series 2017, 884, 012090, doi:10.1088/1742-6596/884/1/012090.

64. Kim, I.H.; Son, J.S.; Min, B.K.; Kim, Y.K.; Kim, K.H.; Kwon, T.Y. A Simple, Sensitive and Non-Destructive Technique for Characterizing Bovine Dental Enamel Erosion: Attenuated Total Reflection Fourier Transform Infrared Spectroscopy. International Journal of Oral Science 2016 8:1 2016, 8, 54-60, doi:10.1038/ijos.2015.58.

65. Delgadoi, A.N.H.S.; Young, A.M. Methacrylate Peak Determination and Selection Recommendations Using ATR-FTIR to Investigate Polymerisation of Dental Methacrylate Mixtures. PLOS ONE 2021, 16, e0252999, doi:10.1371/JOURNAL.PONE.0252999.

66. Sun, L.; Liang, S.; Sa, Y.; Wang, Z.; Ma, X.; Jiang, T.; Wang, Y. Surface Alteration of Human Tooth Enamel Subjected to Acidic and Neutral 30\% Hydrogen Peroxide. Journal of Dentistry 2011, 39, 686-692, doi:10.1016/J.JDENT.2011.07.011.

67. Jaggy, F.; Zinelis, S.; Polychronis, G.; Patcas, R.; Schätzle, M.; Eliades, G.; Eliades, T. ATR-FTIR Analysis and One-Week Stress Relaxation of Four Orthodontic Aligner Materials. Materials 2020, Vol. 13, Page 1868 2020, 13, 1868, doi:10.3390/MA13081868.

68. Barton, P.M.J. A Forensic Investigation of Single Human Hair Fibres Using FTIR-ATR Spectroscopy and Chemometrics. 2011.

69. Wang, Q.; Li, W.; Liu, R.; Zhang, K.; Zhang, H.; Fan, S.; Wang, Z. Human and Non-Human Bone Identification Using FTIR Spectroscopy. International journal of legal medicine 2019, 133, 269-276, doi:10.1007/S00414-018-18228.

70. Leskovar, T.; Zupanič Pajnič, I.; Jerman, I.; Črešnar, M. Separating Forensic, WWII, and Archaeological Human Skeletal Remains Using ATR-FTIR Spectra. International Journal of Legal Medicine 2019, 134, 811-821, doi:10.1007/S00414-019-02079-0/FIGURES/4. 
71. Ciglanská, M.; Jančovičová, V.; Havlínová, B.; Machatová, Z.; Brezová, V. The Influence of Pollutants on Accelerated Ageing of Parchment with Iron Gall Inks. Journal of Cultural Heritage 2014, 15, 373-381, doi:10.1016/J.CULHER.2013.09.004.

72. Legan, L.; Leskovar, T.; Črešnar, M.; Cavalli, F.; Innocenti, D.; Ropret, P. Non-Invasive Reflection FTIR Characterization of Archaeological Burnt Bones: Reference Database and Case Studies. Journal of Cultural Heritage 2020, 41, 13-26, doi:10.1016/j.culher.2019.07.006.

73. Trapp, B.M. The Effects of Household Corrosive Substances on Restored and Non-Restored Teeth, Boston, 2018.

74. Trapp, B.M.; Tallman, S.D. The Effects of Household Corrosive Substances on Silver Amalgam and PorcelainFused-to-Metal Restorations and Non-Restored Teeth. Forensic science international 2018, 293, 77-85, doi:10.1016/J.FORSCIINT.2018.10.004.

75. Vermeij, E.; Zoon, P.; van Wijk, M.; Gerretsen, R. Microscopic Residues of Bone from Dissolving Human Remains in Acids. Journal of forensic sciences 2015, 60, 770-776, doi:10.1111/1556-4029.12700. 